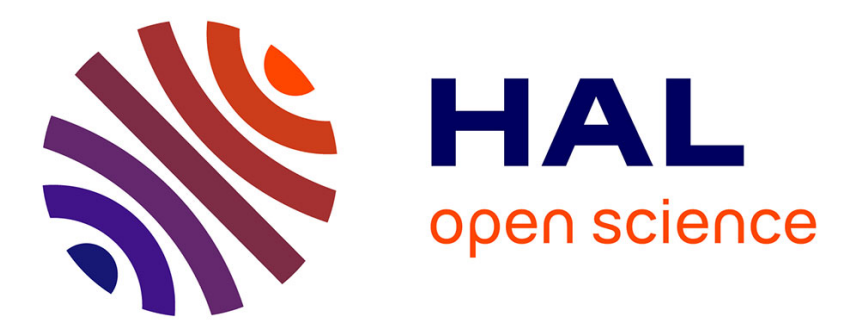

\title{
A Parametric Predictive Maintenance Decision-Making Framework Considering Improved System Health Prognosis Precision
}

Khac Tuan Huynh, Antoine Grall, Christophe Bérenguer

\section{- To cite this version:}

Khac Tuan Huynh, Antoine Grall, Christophe Bérenguer. A Parametric Predictive Maintenance Decision-Making Framework Considering Improved System Health Prognosis Precision. IEEE Transactions on Reliability, 2019, 68 (1), pp.375-396. 10.1109/TR.2018.2829771 . hal-01887627

\section{HAL Id: hal-01887627 https://hal.science/hal-01887627}

Submitted on 4 Oct 2018

HAL is a multi-disciplinary open access archive for the deposit and dissemination of scientific research documents, whether they are published or not. The documents may come from teaching and research institutions in France or abroad, or from public or private research centers.
L'archive ouverte pluridisciplinaire HAL, est destinée au dépôt et à la diffusion de documents scientifiques de niveau recherche, publiés ou non, émanant des établissements d'enseignement et de recherche français ou étrangers, des laboratoires publics ou privés. 


\title{
A Parametric Predictive Maintenance
}

\section{Decision-Making Framework Considering Improved System Health Prognosis Precision}

\author{
K.T. Huynh ${ }^{(*)}$, A. Grall ${ }^{(*)}$ and C. Bérenguer ${ }^{(* *)}$ \\ tuan.huynh@utt.fr,antoine.grall@utt.fr, christophe.berenguer@grenoble-inp.fr \\ ${ }^{(*)}$ ICD, ROSAS, LM2S, Université de Technologie de Troyes, UMR 6281, CNRS, Troyes, France \\ ${ }^{(* *)}$ Univ. Grenoble Alpes, CNRS, Grenoble INP, GIPSA-lab, F-38000 Grenoble, France
}

\begin{abstract}
Health prognosis is an advanced process to forecast the future state of systems, structures and components. Even if it is now recognized as a key enabling step for the maintenance performance improvement on systems and structures, the issue of post-prognosis maintenance decision-making (i.e., how to use prognosis results to eventually make maintenance decisions) remains open. Faced with this situation, we propose a parametric predictive maintenance decision framework that can take into account properly the system remnant life in maintenance decisions. Unlike more classical frameworks, it uses the estimated precision on the prognosis of the system residual useful life as a condition index to decide for and to schedule the interventions on the system. The proposed framework is developed for a single-unit stochastically deteriorating system, maintained through inspection and replacement operations. Using results from the theory of semi-regenerative phenomena, the analytical maintenance cost model is derived for the long-run expected maintenance cost rate. The proposed maintenance decision structure is compared to a classical benchmark framework; numerical experiments evidence the performance and the robustness of the new framework, and confirm the benefit of basing maintenance decisions explicitly on the precision of the system health prognosis (and not only on e.g., the mean value of the estimated residual life).
\end{abstract}

\section{Index Terms}

Condition indices, inspection, analytical cost model, predictive maintenance, prognosis precision, residual useful life, replacement, semi-regenerative theory, stochastic deterioration process.

\section{ACRONYMS}

CBM condition-based maintenance

$\mathrm{CM}$ condition monitoring

RUL residual useful life

MRL mean residual life

pdf probability density function

cdf cumulative distribution function

PR preventive maintenance

CR corrective maintenance

$X_{t}$

$\alpha, \beta$

$m, \sigma^{2}$

$f_{\alpha t, \beta}, F_{\alpha t, \beta}, \bar{F}_{\alpha t, \beta}$

$\Gamma(\cdot), \Gamma(\cdot, \cdot), \Phi(\cdot)$

$L, \tau_{L}, \tau_{i}, \Delta \tau$

$\rho\left(\tau_{i} \mid X_{\tau_{i}}\right), \mu\left(\tau_{i} \mid X_{\tau_{i}}\right)$

$\vartheta\left(\tau_{i} \mid X_{\tau_{i}}\right), \gamma\left(\tau_{i} \mid X_{\tau_{i}}\right)$

$f_{\rho\left(\tau_{i} \mid X_{\tau_{i}}\right)}, R\left(\tau_{i}+u \mid X_{\tau_{i}}\right)$

$\delta, \zeta, \psi$

$\xi$

\section{NOTATIONS}

system deterioration level at time $t$

shape, scale parameters of the homogeneous Gamma deterioration process $\left\{X_{t}\right\}_{t \geq 0}$ average rate, variance rate of $\left\{X_{t}\right\}_{t \geq 0}$

pdf, cdf, survival function of $X_{t}$

complete Gamma function, lower incomplete gamma function, digamma function failure threshold, failure time, $i$-th inspection time, length of a Markov renewal cycle conditional RUL, conditional MRL of the system at $\tau_{i}$ given $X_{\tau_{i}}$ standard deviation, coefficient of variation of $\rho\left(\tau_{i} \mid X_{\tau_{i}}\right)$ pdf of $f_{\rho\left(\tau_{i} \mid X_{\tau_{i}}\right)}$, conditional reliability of the system at $\tau_{i}+u$ given $X_{\tau_{i}}$ inspection period, PR threshold of the $(\delta, \zeta)$ policy, waiting time deterioration threshold associated to prognosis precision 


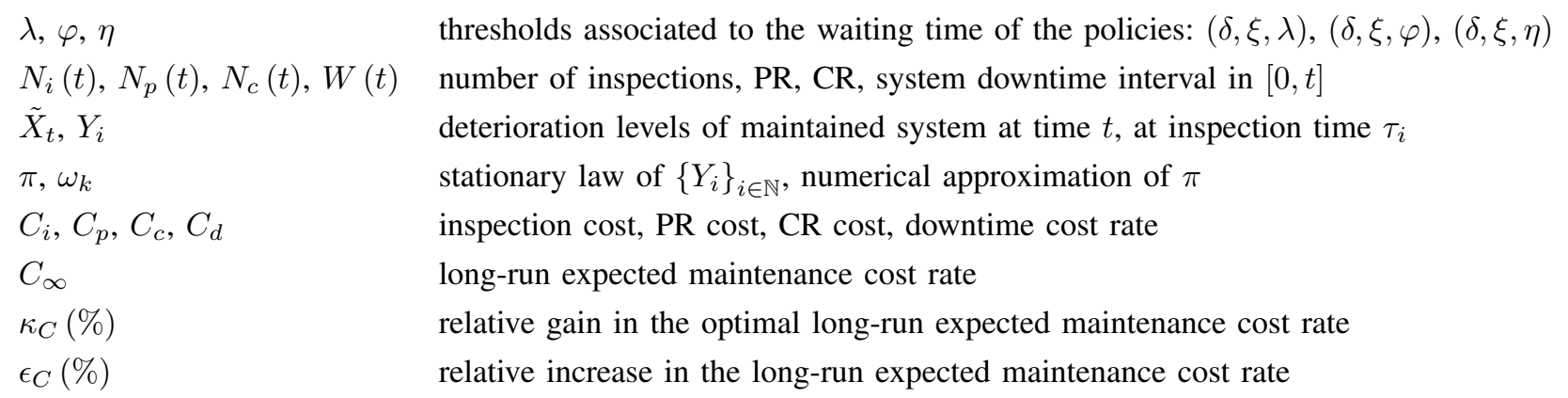

\section{INTRODUCTION}

Maintenance actions include inspection, testing, repair, replacement, that all aim at retaining the maintained system in a proper condition, at improving its availability and extending its life. All these maintenance actions incur costs and they have to be properly organized and scheduled into maintenance policies, built according to a chosen maintenance strategy. In the last decades, maintenance policies have been the object of numerous research works, [1-4]. Chronologically, maintenance strategies have evolved from the naive breakdown or run-to-failure maintenance to preventive maintenance, with first static time-based preventive maintenance, and then condition-based preventive maintenance (CBM) [5]. Recently, with the dissemination of condition monitoring equipments and the development of methods and algorithms for deterioration prognosis and residual life estimation, predictive maintenance becomes possible and attracts more and more interest form practitioners and researchers, [6]. Theoretically, predictive maintenance can be seen as CBM maintenance, but maintenance decisions are based on the online gathered system health prognostic information (i.e., information about the system future state) rather than on the online diagnostic information (i.e., information about the system current state) [7]. Predictive maintenance seeks to anticipate more efficiently system failures in order to plan timely interventions on the system, with the hope of better performance than conventional CBM strategies.

Predictive maintenance models have been extensively proposed over the last few years (see e.g., [8-12] for some recent overviews). Generally, as far as analytical approaches are concerned, predictive maintenance models can be developed according to two main modeling approaches [13, 14]. The first one relies on the (semi)-Markov decision process and dynamic programming tools [15-19], where the system deterioration is assimilated to a multi-state process, and the aim is to find the optimal states to perform predictive maintenance operations. The second one is based on parametric structure-based decision rules and the (semi)-regenerative theory [20-24], where the evolution of system deterioration is considered continuous, and the goal is to determine the set of parameters that tunes the maintenance policy in its optimal configuration. By using the first approach, one is usually faced with two main problems [25]: (i) from a theoretical point of view, it is very difficult and burdensome to formalize and solve the decision problem for a general maintenance policy, even numerically; (ii) from a practical point of view, the resulting maintenance structure of the optimal policy can be quite complex and hard to implement on a real system. Imposing a parametric structure to maintenance decision rules as in the second approach can avoid these drawbacks, since it reduces the size of the problem space when searching for the optimal maintenance policy. Of course, the price paid for this simplification is the risk that the imposed maintenance structure does not correspond to the absolutely optimal policy. However, its feasibility from both theoretical and practical viewpoints leads us to favor the second modeling approach in this paper.

By this second modeling approach, a predictive maintenance policy always has the two common following features [25]:

- condition monitoring scheme. Condition monitoring (CM) can be carried out continuously [26-28] or discretely [29, 30]. By continuous CM, a machine is continuously monitored, and a warning alarm is triggered whenever something wrong is detected. Such a continuous CM approach can be costly, and eventually cannot be implemented in practical engineering applications [5]. In this context, it is more suitable to implement discrete $\mathrm{CM}$ to reveal the system state at inspection times. But of course, with discrete $\mathrm{CM}$, the risk is to miss some failure events occurring between successive inspections. An inspection can be performed statically at fixed CM interval over the whole system life (i.e., periodic inspection) or dynamically at variable CM interval (i.e., non-periodic inspection) [31]. In the second case, unequal CM intervals can either adapt to the system age [32], to the system deterioration rate [33], to the system deterioration level [20, 34], to the system remaining useful life (RUL) [35, 36]), and to the working environment [37], or just be simply random values [38]. From an economic viewpoint, the static inspection schedule is normally less profitable than the dynamic one [31, 36, 39]. However, its implementation in an industrial context is obviously much easier.

- control-limit maintenance decision rule. By this kind of decision rule, maintenance operations are performed on the system whenever the considered condition index characterizing the system health exceeds a critical threshold [2]. In the literature, the most used condition indices are the system deterioration level [29], the system age [39], the system conditional mean residual 
life (MRL) [40], and the system conditional reliability [24]. Based on these indices, we are also able to decide the efficiency of implemented maintenance operations [41]. In reality, a maintenance operation performed on a system may bring it back to an as good as new state (i.e., perfect repair or replacement [42]), to an as bad as old state (i.e., minimal repair [43]), or to an intermediate level between these two states (i.e., imperfect repair [44]). When various maintenance operations are considered, a multi-level control-limit decision rule should be adopted (see e.g., [21, 34, 37, 45]).

These two features (inspection schedule and maintenance decision) are controlled by parameters called decision variables. Optimizing a parametric predictive maintenance policy is then equivalent to tune these decision variables to reach objective functions values. Especially, when the objective function is the asymptotic system unavailability/availability [26, 27, 46], the long-run maintenance cost rate [47-49] or the total discounted cost [50, 51], the optimality of such a maintenance policy has been shown for Markovian deteriorating systems.

In existing works, the decisions on triggering condition monitoring inspections and performing maintenance actions (replacement or repair) are usually linked through the parametric predictive maintenance decision rule. This means that a maintenance operation is always attached to a CM operation, and can be carried out at a certain CM time only [52]. Under this structural property of the maintenance decision rule, some authors have compare predictive maintenance strategies (e.g., reliability-based maintenance strategies, MRL-based maintenance strategies, etc.) and conventional CBM strategies (i.e., deterioration-based maintenance strategies). For instance, Khoury et al. have proposed in [53] two predictive maintenance policies based respectively on maintenance cost and reliability criteria for a gradually deteriorating system operating under uncertain environments. The performance of these policies is evaluated by comparing with a benchmark deterioration-based maintenance policy. In [40], Huynh et al. have quantified the value of health prognostic information for maintenance decision-making by effectuating a comparison between two periodic inspection and replacement policies whose decision parameters are respectively the system MRL and the system deterioration level. The study was done on the basis of a so-called Degradation-Threshold-Dependent-Shock model and using results from the regenerative theory. More recently, a similar study is also considered by Le Son et al. in [54] for a system subject to noisy gamma deterioration process. Other examples can be found in [30, 55-58]. From these works, it is surprising to realize that the performances of the considered maintenance policies are more or less equivalent, and that using (not properly) prognosis information might not make a difference. So, the present paper aims at learning about the reasons for this equivalence, and hence giving some suggestions in order to improve the performances of parametric predictive maintenance strategies. This leads us to seek answers to the two following questions:

1) Is prognostic information really useful for maintenance decision-making and does it bring more information than condition information?

2) If yes, how to properly exploit this prognostic information to make maintenance decision in a consistent and relevant postprognosis maintenance decision framework ?

To this end, from the information about the system deterioration level, we synthesize and analyze various prognostic condition indices associated with the system RUL. Then, we study how these indices should be used in maintenance decision-making.

More precisely, we consider in this work a single-unit deteriorating system subject to inspections returning its deterioration level and replacements. The deterioration and failure behavior of the system is modeled by an univariate homogeneous Gamma process, and the failure occurs at the hitting time of fixed failure threshold. Starting from this model, several prognostic condition indices can be computed, such as the mean value, quantile value, standard deviation, variance and coefficient of variation of the system RUL. Based on these indices, we analyze the current well-known predictive maintenance framework to understand why its performance is limited to the performance of the conventional CBM one. We find out that imposing that "a maintenance operation is always attached to a CM operation, and can be carried out at a certain CM time only" is the main reason for this equivalence. A proper predictive maintenance strategy should be able to get rid of this requirement in order to improve its performance. Following this path, we propose a parametric predictive maintenance decision framework considering improved health prognosis precision. Within this new framework, we distinguish the statistical measures of the variability (i.e. precision) of the system RUL prediction (e.g., standard deviation, variance, coefficient of variation, etc.) from the statistical measures representing the location of the RUL distribution (e.g., mean value, quantile value, etc.). The indices associated with the RUL prediction variability, or prognosis precision, are used to decide whether or not to trigger an inspection on the system, while the indices related to the RUL distribution location are used to determine proper replacement times. Using jointly both these kinds of indices allows us to plan separately inspections and replacements operations, hence avoiding the inherent drawback of the current well-known predictive maintenance framework.

We assess the effectiveness of the new framework by both the performance and robustness. The performance of a maintenance policy is defined as its capacity to save maintenance costs under its optimal configuration. The robustness is its ability to keep the incurred cost close to the optimum one, even when it is not optimally tuned. The performance and robustness of the proposed 
policy are assessed with respect to the long-run expected maintenance cost rate [25]. We develop and optimize the associated mathematical cost model using semi-regenerative theory [59, chapter 10]. Numerical experiments and comparisons under various system configurations (in terms of maintenance costs and deterioration/failure characteristics) show the effectiveness of the proposed maintenance decision framework, and support the benefit of introducing the system health prognostic information in maintenance decision-making.

The paper develops as follows. Section II deals with the system modeling, the computation and analysis of associated condition indices. Section III first motivates the need for a new maintenance decision framework and presents the main assumptions and the original features of the proposed framework integrating the precision on the system health prognosis in the decision rule. Next, the associated mathematical maintenance cost model is derived in Section IV. In Section V, we assess and discuss the performance and the robustness of the new maintenance framework. Conclusions and perspectives for future work close the paper in Section VI.

\section{System Modeling ANd CONDITION INDiCES}

We consider in this section a deterioration-based failure model for a continuously deteriorating single-unit system built on a univariate stochastic deterioration process and a fixed failure threshold. On the basis of this model, we define and compute some well-known diagnostic and prognostic condition indices characterizing the present and future health state of the system. Some properties of these indices essential for maintenance decision-making are also derived and analyzed.

\section{A. System Modeling}

Consider a single-unit (from the maintenance point of view) deteriorating system, subjected to an underlying deterioration process leading eventually to random failures. The deterioration may either result from a physical deterioration phenomenon (cumulative wear, crack growth, erosion, corrosion, fatigue, etc. [34]) or correspond to a loss in performance or a worsening of the system health state with usage and age [60-62]. Modeling the failure process of such a system using classical lifetime models [14] does not allow to reflect properly the deterioration states of the system. Especially, it becomes less relevant in the case of the lack of failure data. To avoid this drawback, we base our system modeling on time-dependent stochastic processes as recommended by Singpurwalla in [63]. The behavior of the system from an as-good-as-new state to a failed state can be captured more finely by a stochastic process, which allows a more accurate prediction of its RUL [64]. We thus represent the deterioration accumulated in the system at time $t \geq 0$ by a scalar random variable $X_{t}$. In the absence of maintenance operation, $X_{t}$ evolves according to an increasing stochastic process $\left\{X_{t}\right\}_{t \geq 0}$ with $X_{0}=0$ (i.e., system new at $t=0$ ). We also assume that the deterioration increment between times $t$ and $s(t \leq s)$, $X_{s}-X_{t}$, is $s$-independent of deterioration levels before $t$. Under these assumptions, any monotone stochastic process belonging to Lévy family [48] can model the system deterioration. Hereinafter, a univariate homogeneous Gamma process with shape parameter $\alpha$ and scale parameter $\beta$ is used. This choice is due to the three following reasons. Firstly, the Gamma deterioration process has been justified by diverse practical applications (e.g., fatigue crack growth [65], carbon-film resistors deterioration [66], corrosion damage mechanism [67], SiC MOSFET threshold voltage deterioration [68], actuator performance loss [69]) and considered appropriate by experts [70]. Secondly, using the homogeneous Gamma process can make the mathematical formulation feasible. And finally, we will see in the following that relying on such an univariate process allows a fair comparison on the performance and robustness of the two parametric predictive maintenance frameworks considered in this paper. Thus, for $t \leq s$, the probability density function (pdf) of deterioration increment $X_{s}-X_{t}$ following a Gamma law is

$$
f_{\alpha \cdot(s-t), \beta}(x)=\frac{1}{\Gamma(\alpha \cdot(s-t))} \beta^{\alpha \cdot(s-t)} x^{\alpha \cdot(s-t)-1} e^{-\beta x} \cdot 1_{\{x \geq 0\}},
$$

and its cumulative distribution function (cdf) is

$$
F_{\alpha \cdot(s-t), \beta}(x)=P\left(X_{s}-X_{t}<x\right)=\frac{\Gamma(\alpha \cdot(s-t), \beta x)}{\Gamma(\alpha \cdot(s-t))},
$$

where $\Gamma(\alpha)=\int_{0}^{\infty} z^{\alpha-1} e^{-z} d z$ is the Gamma function, $\Gamma(\alpha, x)=\int_{0}^{x} z^{\alpha-1} e^{-z} d z$ is the lower incomplete Gamma function, and $1_{\{\cdot\}}$ stands for the indicator function (equal to 1 for a true argument and 0 otherwise). The average deterioration rate is $m=\alpha / \beta$ and variance of the increments is $\sigma^{2}=\alpha / \beta^{2}$. Different values of the parameters $(\alpha, \beta)$ allows to model different kinds and different variabilities of deterioration behaviors. A review of various statistical methods for the estimation of $(\alpha, \beta)$ from deterioration data can be found in [8].

Based on this deterioration process, a deterioration-threshold failure is considered [71]. In practice, for safety or economic reasons (e.g., high risk of hazardous breakdowns, poor products quality, high ressources consumption), a system is considered as failed when it is too worn, even if it is still functioning. Accordingly, we consider that the system fails when its deterioration hits a critical 
prefixed threshold $L$. Such a failure can be either an actual hard failure of an active system, or a pending failure of a passive system or structure. The random failure time of the system $\tau_{L}$ is

$$
\tau_{L}=\inf \left\{t \in \mathbb{R}^{+} \mid X_{t} \geq L\right\}
$$

Note that by this model, we only consider the deterioration-dependent failure mode, all failures not directly related to the system deterioration are discarded.

\section{B. Condition indices}

Condition indices are usually the results returned by the real-time diagnosis of impending failures (i.e., diagnostic condition indices) or by the prognosis of future system health (i.e., prognostic condition indices) [72]. They characterize the current or future health state of a system, and hence provide useful information for maintenance-decision making [40]. In our case, we take as a diagnostic condition index the deterioration level $X_{\tau_{i}}$ revealed by an inspection at time $\tau_{i}$, since it defines the system health state at the current time $\tau_{i}$. Of course, diagnosing a system can be a complex task and usually requires advanced techniques [73, 74], and diagnosis results may also be affected by noise and disturbances [75]. However, diagnosis methods are beyond the scope of this work. We simply consider that an inspection diagnoses and reveals perfectly the system deterioration level. Based on the diagnostic information, prognostic condition indices can also be evaluated using the deterioration-based failure model. The system RUL, defined as the duration until the end of the system useful life [76], carries most of the prognostic information, as shown in the literature. It is a random variable from which various prognostic condition indices can be derived: its mean value, its quantile values, its standard deviation, its variance and its coefficient of variation. The concept of RUL has been the object of many works (see e.g., [77-79]). Here, as in [80], we consider a so-called deterioration-based RUL, defined at time $\tau_{i}$ as a conditional random variable given the deterioration level $X_{\tau_{i}}$ [64] :

$$
\rho\left(\tau_{i} \mid X_{\tau_{i}}\right)=\left(\tau_{L}-\tau_{i} \mid X_{\tau_{i}}\right) \cdot 1_{\left\{X_{\tau_{i}}<L\right\}}
$$

where $\tau_{L}$ is the system failure time in (3). For $X_{\tau_{i}}<L$, the survival function of the conditional RUL $\rho\left(\tau_{i} \mid X_{\tau_{i}}\right)$ is

$$
P\left(\rho\left(\tau_{i} \mid X_{\tau_{i}}\right)>u\right)=P\left(\tau_{L}-\tau_{i}>u \mid X_{\tau_{i}}\right)=P\left(\tau_{L}>\tau_{i}+u \mid X_{\tau_{i}}\right)=R\left(\tau_{i}+u \mid X_{\tau_{i}}\right)
$$

where $R\left(\tau_{i}+u \mid X_{\tau_{i}}\right)$ denotes the conditional reliability of the system at a future time $\tau_{i}+u$ given the deterioration level $X_{\tau_{i}}$ at the current time $\tau_{i}$. The expression (5) indicates that the survival function of the system RUL at a current time is equivalent to the corresponding system reliability at a future time. This explains why the RUL information is suitable for predictive maintenance planning and decision-making. Knowing $X_{\tau_{i}}=x$, this conditional reliability can be computed by

$$
R\left(\tau_{i}+u \mid X_{\tau_{i}}=x\right)=P\left(X_{\tau_{i}+u}<L \mid X_{\tau_{i}}=x\right)=P\left(X_{\tau_{i}+u}-X_{\tau_{i}}<L-x\right)=F_{\alpha u, \beta}(L-x),
$$

where $F_{\alpha u, \beta}(\cdot)$ is derived from (2). The associated pdf is then [29]

$$
f_{\rho\left(\tau_{i} \mid X_{\tau_{i}}=x\right)}(u)=-\frac{\partial}{\partial u} F_{\alpha u, \beta}(L-x)=\frac{\alpha}{\Gamma(\alpha u)} \int_{\beta \cdot(L-x)}^{\infty}(\ln (z)-\Phi(\alpha u)) z^{\alpha u-1} e^{-z} d z,
$$

where $\Phi(v)=\frac{\partial}{\partial v} \ln (\Gamma(v))$ is known as the digamma function. Figures 1a and $1 \mathrm{~b}$ illustrate the survival function and the pdf of the conditional RUL for the system defined by the set of parameters $L=15,(\alpha, \beta)=(1 / 3,1 / 3)$ (i.e., small variance: $\sigma^{2}=3$ ) and $(\alpha, \beta)=(1 / 9,1 / 9)$ (i.e., high variance: $\left.\sigma^{2}=9\right)$ respectively. It is easy to prove the monotony of $R\left(\tau_{i}+u \mid x\right)$ [40]: for $a$ fixed $u$, where $u \geq 0, R\left(\tau_{i}+u \mid x\right)$ is non-increasing in $x$; for a fixed $x$, where $0 \leq x<L, R\left(\tau_{i}+u \mid x\right)$ is non-increasing in $u$. This property leads to higher slope in $R\left(\tau_{i}+u \mid x\right)$ for a higher value of $x$, hence the narrower probability density function of the conditional RUL. Moreover, $R\left(\tau_{i}+u \mid x\right)$ is less steep when $\sigma^{2}$ is high, hence the broader probability density function of the conditional RUL. These phenomena are shown clearly in Figures 1a and 1b. In short, the RUL prognosis becomes more precise for a higher value of $x$ and smaller value of $\sigma^{2}$. This property is very important in developing the new predictive maintenance decision framework (see also Section III-D).

To characterize the cdf of the random system RUL $\rho\left(\tau_{i} \mid X_{\tau_{i}}\right)$, statistical quantities such as its mean value, its standard deviation and its coefficient of variance are used. The mean value of the system RUL, commonly known as the system MRL, indicates the location of the RUL cdf. Let $\mu\left(\tau_{i} \mid X_{\tau_{i}}\right)$ denote the system conditional MRL at time $\tau_{i}$ given the deterioration level $X_{\tau_{i}}$; when $X_{\tau_{i}}=x$, it is computed by

$$
\mu\left(\tau_{i} \mid X_{\tau_{i}}=x\right)=E\left[\rho\left(\tau_{i} \mid X_{\tau_{i}}=x\right)\right]=\int_{0}^{\infty} F_{\alpha u, \beta}(L-x) d u
$$




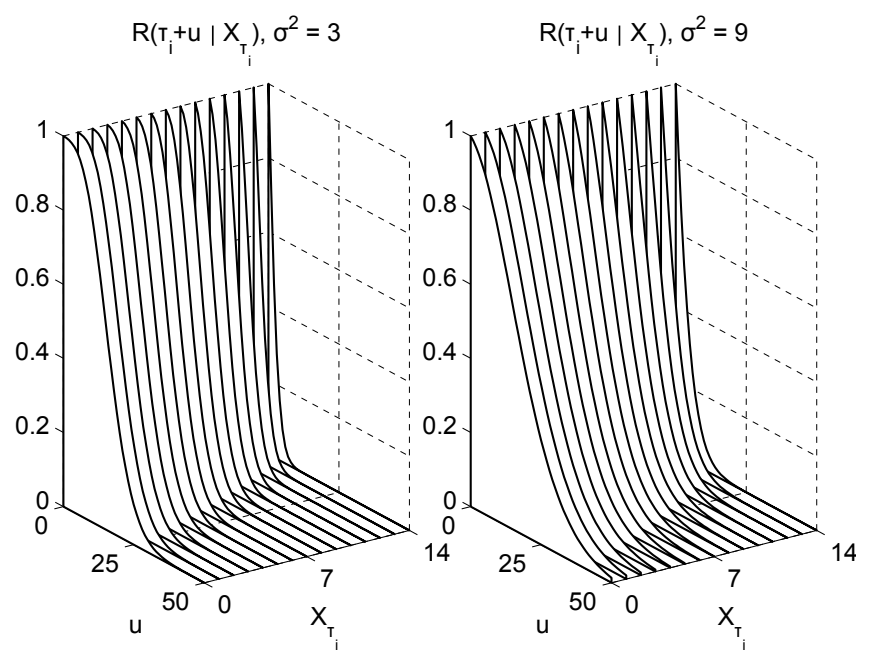

(a) Survival function of $\rho\left(\tau_{i} \mid X_{\tau_{i}}\right)$

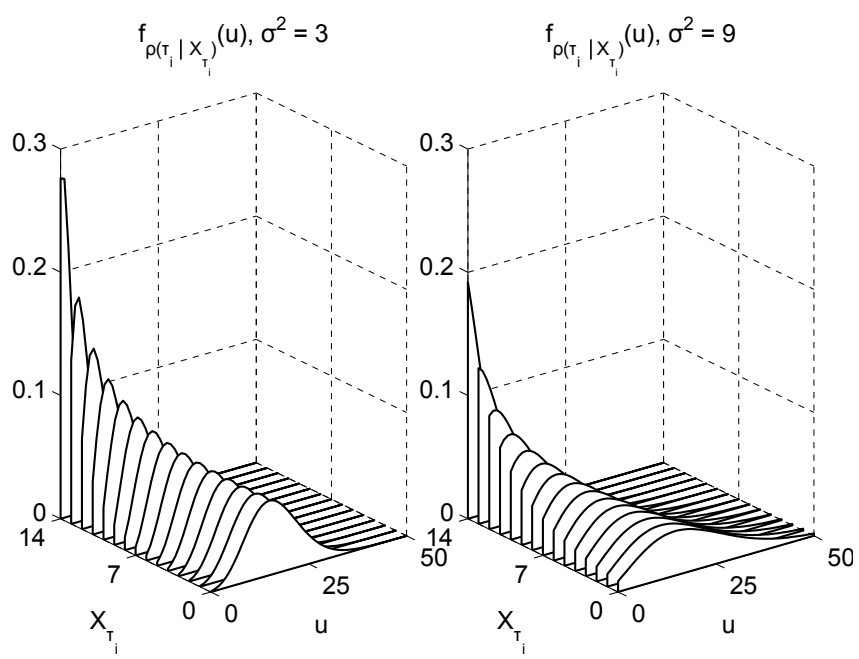

(b) Pdf of $\rho\left(\tau_{i} \mid X_{\tau_{i}}\right)$

Figure 1: Probability law of the system conditional RUL $\rho\left(\tau_{i} \mid X_{\tau_{i}}\right)$

The standard deviation and coefficient of variance of the system RUL are adopted to characterize its variability. While the standard deviation often measures the spread or dispersion of $\rho\left(\tau_{i} \mid X_{\tau_{i}}\right)$, the coefficient of variance measures its scale invariant dispersion because the conditional MRL is taken into account. Let $\vartheta\left(\tau_{i} \mid X_{\tau_{i}}\right)$ be the standard deviation of $\rho\left(\tau_{i} \mid X_{\tau_{i}}\right)$; when $X_{\tau_{i}}=x$, it is computed by

$$
\vartheta\left(\tau_{i} \mid X_{\tau_{i}}=x\right)=\sqrt{2 \int_{0}^{\infty} u F_{\alpha u, \beta}(L-x) d u-\mu^{2}\left(\tau_{i} \mid X_{\tau_{i}}=x\right)} .
$$

The proof of (9) is shown in Appendix A. One can derive from (8) and (9) the associated coefficient of variation $\gamma\left(\tau_{i} \mid X_{\tau_{i}}=x\right)$ as

$$
\gamma\left(\tau_{i} \mid X_{\tau_{i}}=x\right)=\frac{\vartheta\left(\tau_{i} \mid X_{\tau_{i}}=x\right)}{\mu\left(\tau_{i} \mid X_{\tau_{i}}=x\right)}=\sqrt{2 \frac{\int_{0}^{\infty} u F_{\alpha u, \beta}(L-x) d u}{\int_{0}^{\infty} F_{\alpha u, \beta}(L-x) d u}-1 .}
$$

Recall that in (8), (9) and (10), $F_{\alpha u, \beta}(\cdot)$ is given by (2). For an illustration, we sketch the shape of the conditional MRL $\mu\left(\tau_{i} \mid X_{\tau_{i}}=x\right)$, the standard deviation $\vartheta\left(\tau_{i} \mid X_{\tau_{i}}=x\right)$ and the coefficient of variation $\gamma\left(\tau_{i} \mid X_{\tau_{i}}=x\right)$ in Figure 2 when $x$ varies. One can remark from (8), (9) and (10) that these quantities depend only on $x$. Thus, given the deterioration-based failure model as in
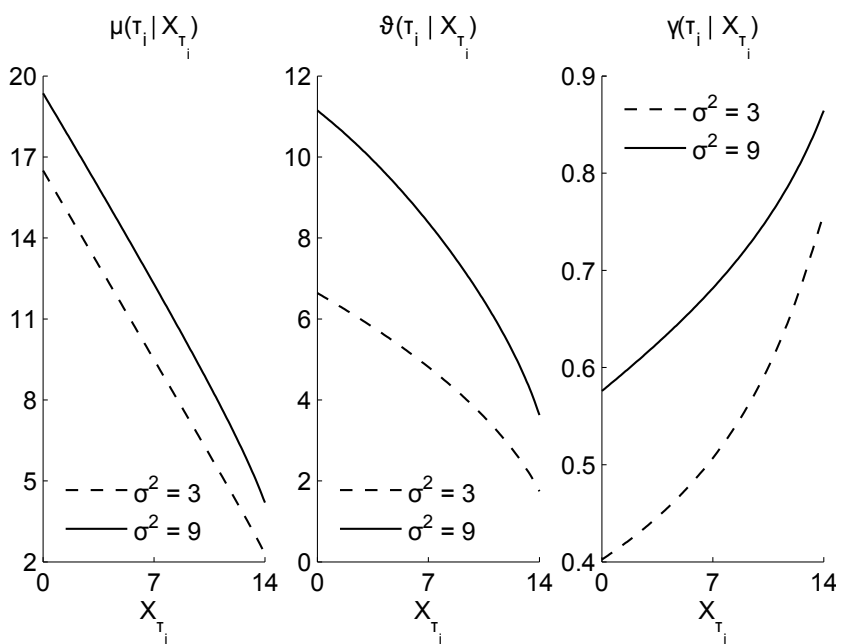

Figure 2: Statistical quantities characterizing the cdf of the conditional RUL $\rho\left(\tau_{i} \mid X_{\tau_{i}}\right)$

Section II-A, the diagnostic and prognostic condition indices are equivalent. Furthermore, we can easily show that $\mu\left(\tau_{i} \mid X_{\tau_{i}}=x\right)$ and $\vartheta\left(\tau_{i} \mid X_{\tau_{i}}=x\right)$ are non-increasing in $x$ and equal to 0 when $x \geq L$, while $\gamma\left(\tau_{i} \mid X_{\tau_{i}}=x\right)$ is non-decreasing in $x$ and its value belongs to the interval $[0,1]$. Although we can transform the above diagnostic and prognostic condition indices from one to each other (i.e., transform $X_{\tau_{i}}=x$ to $\mu\left(\tau_{i} \mid x\right)$, to $\vartheta\left(\tau_{i} \mid x\right)$ or to $\gamma\left(\tau_{i} \mid x\right)$, and vice versa), each of them has its own meaning. A proper maintenance policy should take care of this point. In Section III-D, such maintenance policies will be proposed.

The conditional reliability $R\left(\tau_{i}+u \mid x\right)$ and the conditional MRL $\mu\left(\tau_{i} \mid x\right)$ of the system will be adopted to make predictive 
replacement decisions adaptive to the system deterioration level. So, it is interesting to study the sensitivity of these indices with respect to the system deterioration variability. To this end, we take the derivative of $R\left(\tau_{i}+u \mid x\right)$ and $\mu\left(\tau_{i} \mid x\right)$ according to $x$

$$
\frac{\partial R\left(\tau_{i}+u \mid x\right)}{\partial x}=\frac{\partial F_{\alpha u, \beta}(L-x)}{\partial x}=-f_{\alpha u, \beta}(L-x)
$$

and

$$
\frac{d \mu\left(\tau_{i} \mid x\right)}{d x}=\frac{d\left(\int_{0}^{\infty} F_{\alpha u, \beta}(L-x) d u\right)}{d x}=-\int_{0}^{\infty} f_{\alpha u, \beta}(L-x) d u,
$$

$f_{\alpha u, \beta}(\cdot)$ is given by (1). Setting $L=15$, the shape of $\left|\partial R\left(\tau_{i}+u \mid x\right) / d x\right|$ and $\left|d \mu\left(\tau_{i} \mid x\right) / d x\right|$ with respect to $x$ are shown in Figure 3 when the values of the deterioration variance rates are $\sigma^{2}=3$ and 9 respectively. Obviously, $\left|d \mu\left(\tau_{i} \mid x\right) / d x\right| \geq$

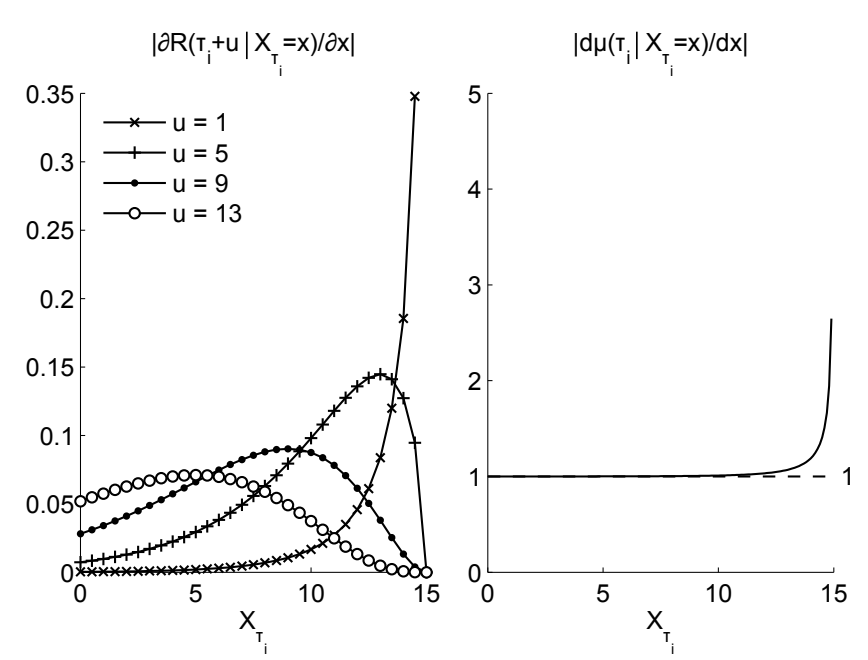

(a) $(\alpha, \beta)=(1 / 3,1 / 3)$ (i.e., small variance: $\left.\sigma^{2}=3\right)$

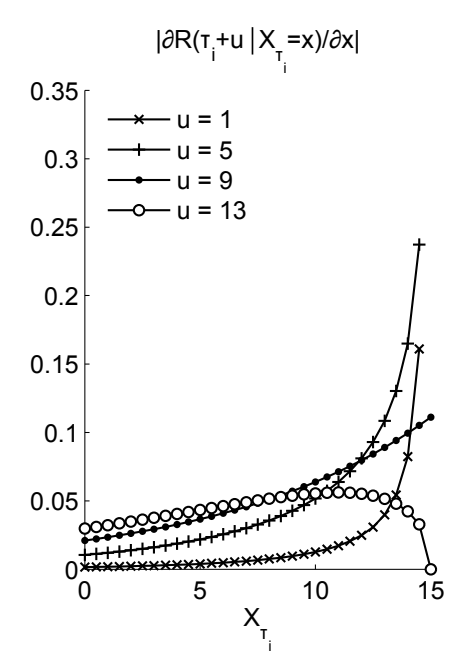

(b) $(\alpha, \beta)=(1 / 9,1 / 9)$ (i.e., high variance: $\left.\sigma^{2}=9\right)$

Figure 3: Derivative of $R\left(\tau_{i}+u \mid x\right)$ and $\mu\left(\tau_{i} \mid x\right)$ according to $x$

$\left|\partial R\left(\tau_{i}+u \mid x\right) / d x\right|$, which means that the system conditional MRL is thus more sensitive to the variability of the system deterioration than the system conditional reliability. Moreover, when the variance of the deterioration process $\sigma^{2}$ increases, $\left|\partial R\left(\tau_{i}+u \mid x\right) / d x\right|$ decreases while $\left|d \mu\left(\tau_{i} \mid x\right) / d x\right|$ increases (see Figure 3a vs. Figure 3b); this sensitivity is thus even more important for higher $\sigma^{2}$. Consequently, the system conditional reliability is less sensitive to the variability of deterioration than the system conditional MRL.

\section{Parametric Predictive Maintenance Decision Frameworks}

A predictive maintenance decision framework taking into account the precision on the system health prognosis is proposed in this section. It relies on the parametric decision rule presented in [25]. We first introduce our hypotheses on the maintained system. Then, we present and analyze in detail a maintenance policy stemming from a classical "state-of-the-art" maintenance decision framework. Next, we introduce our maintenance decision framework. Finally, within this new framework, three predictive maintenance policies are derived and their behavior is investigated in detail using numerical experiments.

\section{A. General Maintenance Assumptions}

Consider the single-unit system presented in Section II-A, we assume that the deterioration level of the system is hidden, and its failure state is non-self-announcing. This means that the system reveals only its deterioration level and its failure/working state through a monitoring procedure. Continuous monitoring allows observing the system state continuously. However, it is usually very expensive and sometimes impossible in practical engineering applications [37]. It is therefore more suitable to implement inspection operations at discrete times. Recall that, the inspection operation mentioned here stands for the system diagnosis. It is not simply the data collection as usual, but also the feature extraction from the collected data, the construction of deterioration indicators, and perhaps more [81]. In some ways, we can consider it as all the tasks before the Maintenance Decision Making task in a traditional CBM program [7]. Such an inspection operation is itself costly, and takes time. However, the time for an inspection is still negligible compared to the life cycle of a system. Thus, we can assume that each inspection operation is non-destructive, perfect, instantaneous, and incurs a cost $C_{i}>0$.

Two maintenance actions are possible on the system : preventive replacement $(\mathrm{PR})$ and corrective replacement (CR) which respectively incur a cost $C_{p}>C_{i}$ and $C_{c}$. These replacements are true physical replacements (and not repairs) that make the system 
as-good-as-new. Their duration is neglected, and it is assumed that they incur fixed costs, irrespective of the deterioration level of the system. Moreover, the maintenance costs are not necessarily identical in practice even though both PR and CR returns the system to an as-good-as-new condition. It is due to the fact a CR is not scheduled in advance and is performed on a more deteriorated system. The cost $C_{c}$ also includes different costs associated to the failure consequences, due e.g., to damage to the environment. A CR is thus likely to be more complex and more expensive than a PR (i.e., $C_{c}>C_{p}$ ). Furthermore, a replacement, whether preventive or corrective, can only be instantaneously performed at predetermined times (i.e., inspection time or scheduled replacement time) and the system downtime $W(\cdot)$ after failure until the next replacement incurs a cost at rate $C_{d}$.

The maintenance costs $C_{i}, C_{p}$ and $C_{c}$ are given in cost unit, while $C_{d}$ is given in cost unit/time unit.

\section{B. Maintenance Cost Performance Criterion}

The performance of the considered policies is assessed using the long-run expected maintenance cost rate. As in [25], the long-run expected maintenance cost rate is defined by

$$
C_{\infty}=\lim _{t \rightarrow \infty} \frac{E[C(t)]}{t},
$$

where $C(t)$ is the total maintenance cost including the downtime cost up to time $t$. According to the general maintenance assumptions given in Section III-A, $C(t)$ is expressed as

$$
C(t)=C_{i} \cdot N_{i}(t)+C_{p} \cdot N_{p}(t)+C_{c} \cdot N_{c}(t)+C_{d} \cdot W(t),
$$

where $N_{i}(t), N_{p}(t)$ and $N_{c}(t)$ are respectively the number of inspections, PR and CR operations in $[0, t], W(t)$ is the system downtime interval in $[0, t]$. For a given parametric maintenance policy, the behavior of the maintained system and $C_{\infty}$ depend obviously on the values of the policy decision variables. The mathematical model to evaluate $C_{\infty}$ is derived in Section IV, which allows to optimize the considered maintenance policies by tuning their decision variables at their optimal values. In the present section, numerical experiments are presented for a system characterized by the set of parameters $\alpha=1 / 3, \beta=1 / 3, L=15$ and for the maintenance costs $C_{i}=5, C_{p}=50, C_{c}=100$ and $C_{d}=25$. For a sound and meaningful analysis and comparison of the behavior of the proposed maintenance schemes, the numerical experiments are performed with the optimal decision variables obtained for each of the considered maintenance policies with the cost model presented in Section IV.

\section{Current Predictive Maintenance Decision Framework \& Representative Policy}

As already mentioned in Section I, within the current well-known predictive maintenance decision frameworks (see e.g., [5, 8, 52]), a replacement operation is always linked to an inspection and can thus be triggered only at an inspection time $\tau_{i}$. Most maintenance policies presented in published works share this characteristic. For instance, in [29, 82], periodic inspection and replacement policies are proposed, whose replacement operations are performed when the inspected deterioration level first exceeds a given threshold. In $[20,33,34,83]$, in the same way, the deterioration level is used to make a replacement decision for non periodic inspections. In [24] and [40], the replacement decisions are made at inspection times according to the system conditional reliability and the system conditional MRL respectively. Many works in the literature show that this predictive maintenance framework is relatively effective for single-unit systems subject to multiple failure modes (i.e. due to either shocks or deterioration) or for multi-unit systems. This can be explained by the "overarching" property of prognostic condition indices (e.g., the system reliability, the system MRL, the standard deviation or the coefficient of variance of the system RUL, etc.), and a prognosis-based maintenance decision-making is more profitable than a diagnosis-based maintenance decision-making (i.e. based on the system deterioration level) [40], [24]. However, when considering a single-unit system subject to a single failure mode only due to deterioration, using a prognostic or a diagnosis condition index within a classical predictive maintenance decision framework leads to almost similar performances [40, 54]. Indeed, this framework does not give the opportunity to use fully the prognostic information to reach better maintenance performance. In the following, we study a representative maintenance policy stemming from this framework to investigate and analyze the reasons behind this behavior.

For a better understanding, a periodic inspection scheme with period $\delta$ is assumed, and the replacement decisions are made according to the detected deterioration level of the system at an inspection time $\tau_{i}$. Of course, other inspection schemes (e.g., quantile-based inspection, random inspection, etc.) and other condition indices for maintenance decision-making (e.g., system reliability, system MRL, coefficient of variance of the system RUL, etc.) could be implemented at the price of a more difficult (even intractable) analytical formulation. Let define the time interval between two successive replacement operations as a renewal cycle of the system. Consider the following policy with periodic inspection and deterioration-based replacement : 
1) The system is periodically inspected with period $\delta>0$.

2) At a planned inspection date $\tau_{i}=i \cdot \delta, i=1,2, \ldots$, a system found failed (i.e., $X_{\tau_{i}} \geq L$ ) is correctively replaced. After the replacement, the system becomes as-good-as-new, and a new renewal cycle begins. The next inspection time is scheduled on this new cycle at $\tau_{i+1}=\tau_{i}+\delta$.

3) If the system is still running at $\tau_{i}$, an additional decision based on the deterioration level $X_{\tau_{i}}$ is made.

- If $\zeta \leq X_{\tau_{i}}<L$, the running system is considered too worn, and the system is preventively replaced at $\tau_{i}$. After the replacement, the system is as-good-as-new, and a new renewal cycle starts. The next inspection time is scheduled on this new cycle at $\tau_{i+1}=\tau_{i}+\delta$.

- Otherwise, nothing is done at $\tau_{i}$, and the maintenance decision is postponed at the next inspection time at $\tau_{i+1}=\tau_{i}+\delta$.

The inspection period $\delta$ and the PR threshold $\zeta$ are the two decision variables for this policy, called $(\delta, \zeta)$ policy.
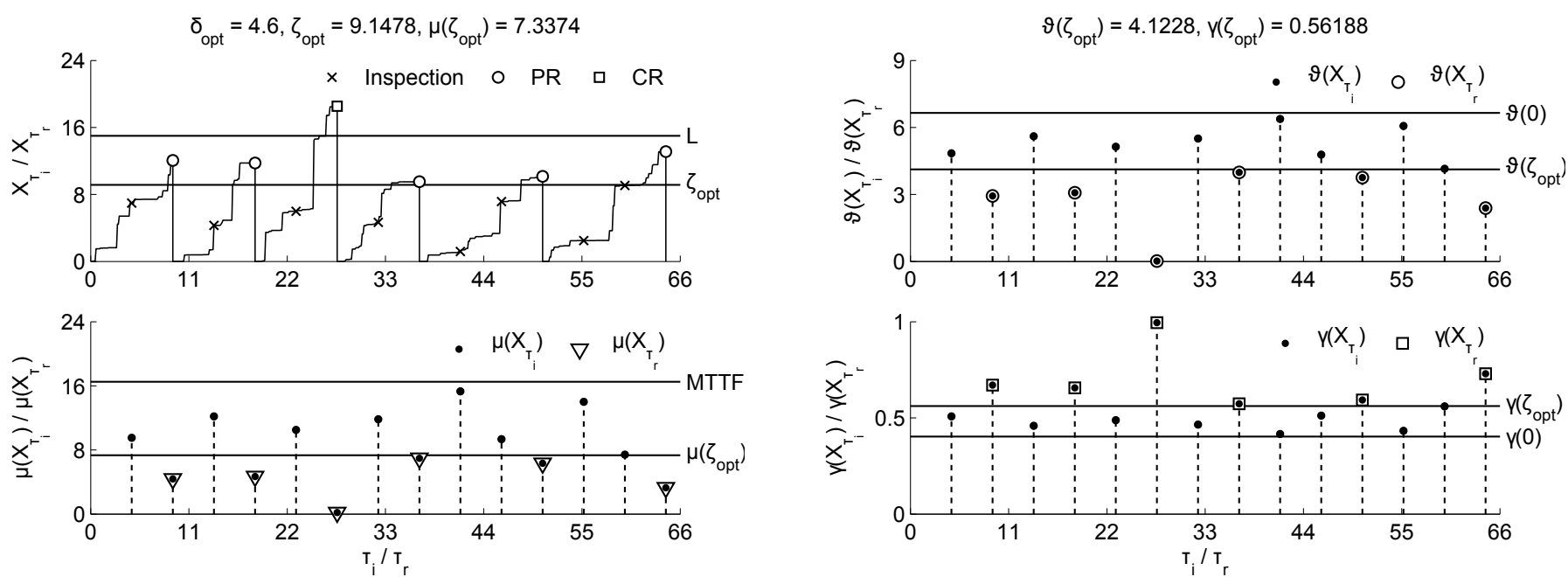

(a) Evolution of $X_{\tau_{i}} \mid X_{\tau_{r}}$ and $\mu\left(X_{\tau_{i}}\right) \mid \mu\left(X_{\tau_{r}}\right)$

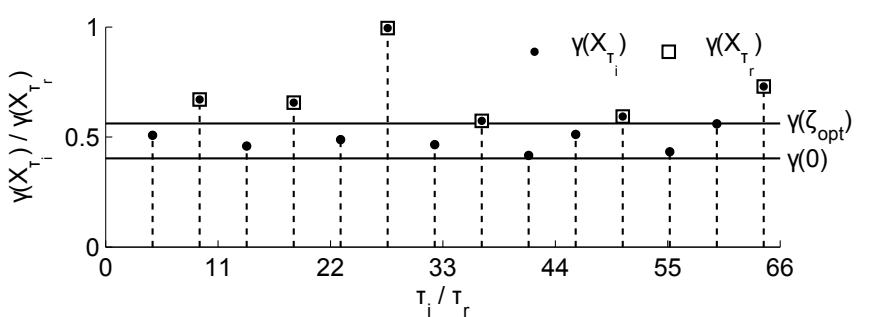

(b) Evolution of $\vartheta\left(X_{\tau_{i}}\right) \mid \vartheta\left(X_{\tau_{r}}\right)$ and $\gamma\left(X_{\tau_{i}}\right) \mid \gamma\left(X_{\tau_{r}}\right)$

Figure 4: Illustration of the $(\delta, \zeta)$ policy

For a gradually deteriorating system as described in Section II-A, with the parameters values given in Section III-B, the optimal decision variables of the $(\delta, \zeta)$ policy (obtained through an optimization procedure with the cost model developed in Section IV) are $\delta_{\text {opt }}=4.6$ and $\zeta_{\text {opt }}=9.1478$. Figure 4 illustrates the behavior the maintained system for this optimal tuning $: \tau_{i}$ and $\tau_{r}$ represent the inspection times and replacement times respectively. The deterioration paths of the maintained system and the associated inspection and replacement operations are shown on the top of Figure 4a. The evolution of the 3 prognostic condition indices: conditional MRL, the standard deviation and the coefficient of variation of the conditional RUL at inspection and replacement times are also represented in the bottom of Figure $4 \mathrm{a}$ and in Figure $4 \mathrm{~b}$.

Analyzing the $(\delta, \zeta)$ policy, we find that the threshold $\zeta$ has a double role: (i) deciding whether or not to trigger a PR; (ii) deciding, together with the inspection period $\delta$, for the number of inspections carried out over a renewal cycle. In our opinion, this could be the biggest weakness of the $(\delta, \zeta)$ policy, as well as of the classical "state-of-the-art" predictive maintenance decision framework. Indeed, a single threshold $\zeta$ might not be enough to make efficient decision in order to both avoid inopportune inspections and properly prolong the system useful lifetime. To avoid a large number of inspections, the $(\delta, \zeta)$ policy should be tuned with a low $\zeta$; however a low $\zeta$ shortens the system useful lifetime because of early PR operations. On the contrary, a high value of $\zeta$ can lengthen the system useful lifetime, but leads to an increased cumulative inspection cost due to unnecessary redundant inspections. We should handle this weakness to improve the effectiveness of the current predictive maintenance decision framework, and adding more degrees of freedom could help to make more adapted maintenance decisions.

\section{Predictive Maintenance Decision Framework Based on Improved Health Prognosis Precision \& Associated Policies}

To remedy the drawback of the more classical predictive maintenance decision framework, we propose to use different condition indices for replacement decision and inspections scheduling. As such, two condition indices instead of a single one should be resorted to: one for control of replacement decision, and the other for control of inspection decision. Accordingly, it is not necessary to always attach a replacement at a given inspection time $\tau_{i}$, but rather at a predetermined time $\tau_{r}$ (normally $\tau_{r} \neq \tau_{i}$ ). Moreover, to do or not to do an additional inspection on the system could be based on the precision of prognostic information of the system health state. In fact, since the system health prognosis precision can improve itself with age (review Section II-B), from a certain age $\tau_{i}$ of the 
system, its failure time can be estimated with high precision. Thus, additional inspection operations are no longer necessary, and we just wait $\psi$ time units, $\psi \geq 0$, from the last inspection to do a system replacement at $\tau_{r}=\tau_{i}+\psi$. This is the main principle of our new predictive maintenance decision framework. Obviously, the use of two different health indices for the replacement decision and inspection scheduling makes it more flexible than the traditional framework. Furthermore, the new framework can also return to the traditional one when the waiting time $\psi=0$, hence it is more general and more profitable in most cases. In other words, there is no risk in using this proposed parametric predictive maintenance decision framework instead of the traditional one. In the following, a representative maintenance decision rule stemming from this new framework is introduced for illustration purposes.

Normally, the standard deviation or coefficient of variance of the conditional system RUL can be used as measures of the precision of the health prognosis. For our considered system model (Section II-A), the deterioration level detected at an inspection time returns the same information as the standard deviation or coefficient of variance of the conditional system RUL: the higher the deterioration, the more the system RUL prognosis is precision. So, we merely use the system deterioration level to control inspection decisions. This choice, on one hand, simplifies the computation, and on other hand, allows maintenance decision rules consistent with the aforementioned representative of the current well-known predictive maintenance decision framework. Of course, the choice is only valid for single-unit systems subject to univariate stochastic deterioration process as our considered system. When the system becomes more complex (e.g., multi-failure mode or multi-unit), the more "overarching" variance or coefficient of variance of the conditional system RUL should be used instead of the simple system deterioration level. About the waiting time $\psi$, it can be determined from the system conditional MRL and the system conditional reliability. As a result, we can state a typical and exemplary maintenance decision rule as follows.

1) Over a given renewal cycle, we regularly inspect the system with period $\delta$.

2) At a planned inspection date $\tau_{i}=i \cdot \delta, i=1,2, \ldots$, a $\mathrm{CR}$ is performed on the system if it fails (i.e., $X_{\tau_{i}} \geq L$ ). After the replacement, the system becomes as-good-as-new, and a new renewal cycle begins. The next inspection time is scheduled on this new cycle at $\tau_{i+1}=\tau_{i}+\delta$.

3) If the system is still running, we define $\xi$ as a deterioration threshold indicating the precision of the system RUL prognosis, and we adopt the following decision rules.

- If $\xi \leq X_{\tau_{i}}<L$, we cannot predict the system failure time with an acceptable precision, so no additional inspection is needed for the current renewal cycle, and a system replacement will be performed $\psi$ time units later (i.e., at time $\left.\tau_{r}=\tau_{i}+\psi\right)$. The replacement at $\tau_{r}$ may be either preventive or corrective depending on the working or failure state of the system at this time. After the replacement, the system becomes as-good-as-new, and a new renewal cycle begins. The next inspection will be done on the new cycle at $\tau_{i+1}=\tau_{r}+\delta=\tau_{i}+\psi+\delta$.

- Otherwise (i.e., $X_{\tau_{i}}<\xi$ ), the system failure time can be precisely estimated, so we need to do one or more inspections to gather additional information about the system. The maintenance decisions are thus postponed until the next inspection time $\tau_{i+1}=\tau_{i}+\delta$.

We can remark that the waiting time $\psi$ brings about the main difference between this maintenance decision rule and the representative of the traditional framework. How to build such a waiting time is obviously very important because it influences significantly the maintenance cost saving and the robustness of the new framework. Hereafter, we will discuss more deeply this issue through three predictive maintenance policies: $(\delta, \xi, \lambda),(\delta, \xi, \varphi)$ and $(\delta, \xi, \eta)$.

1) $(\delta, \xi, \lambda)$ policy: This policy is derived from the above maintenance decision rule where the waiting time $\psi$ is built from a time-based approach: $\psi$ is defined as a constant time interval $\lambda$ (i.e., $\psi \triangleq \lambda$ ) irrespective of the system state at latest inspection date. The inspection period $\delta$, the deterioration threshold to control prognosis precision $\xi$, and the waiting time interval $\lambda$ are the 3 decision variables to be optimized, and hence this policy is called $(\delta, \xi, \lambda)$. Using the full mathematical cost model in Section IV to optimize the $(\delta, \xi, \lambda)$ policy for a deteriorating system (described in Section II-A, with the parameters values given in Section III-B), one obtains the optimal tuning $\delta_{o p t}=5.4, \xi_{\text {opt }}=7.3502$ and $\lambda_{o p t}=1.2$. The corresponding behavior of the maintained system is shown in Figures $5 \mathrm{a}$ and $5 \mathrm{~b}$. The meanings of these figures are the same as the ones of the $(\delta, \zeta)$ policy illustrated in Section III-C. We can see that the optimal inspection period $\delta_{\text {opt }}$ of the $(\delta, \xi, \lambda)$ policy is longer than the one of the $(\delta, \zeta)$ policy. Moreover $\xi_{\text {opt }}<\zeta_{\text {opt }}$, which means that the optimal $(\delta, \xi, \lambda)$ policy does not need a RUL prediction precision as high as in the optimal $(\delta, \zeta)$ policy. In other words, the information about the health prognosis precision has been taken into account to improve the maintenance decision-making, and the $(\delta, \xi, \lambda)$ policy can save inspection costs. While the time-based $\psi$ building approach is easy to implement, it still suffers the weakness that the system replacement might be too early (e.g., first replacement in Figure 5a) or too late (e.g., second replacement in Figure 5a), because $\psi$ is scheduled regardless of the system condition. To avoid this drawback, one should resort to condition-based $\psi$ building approach. 

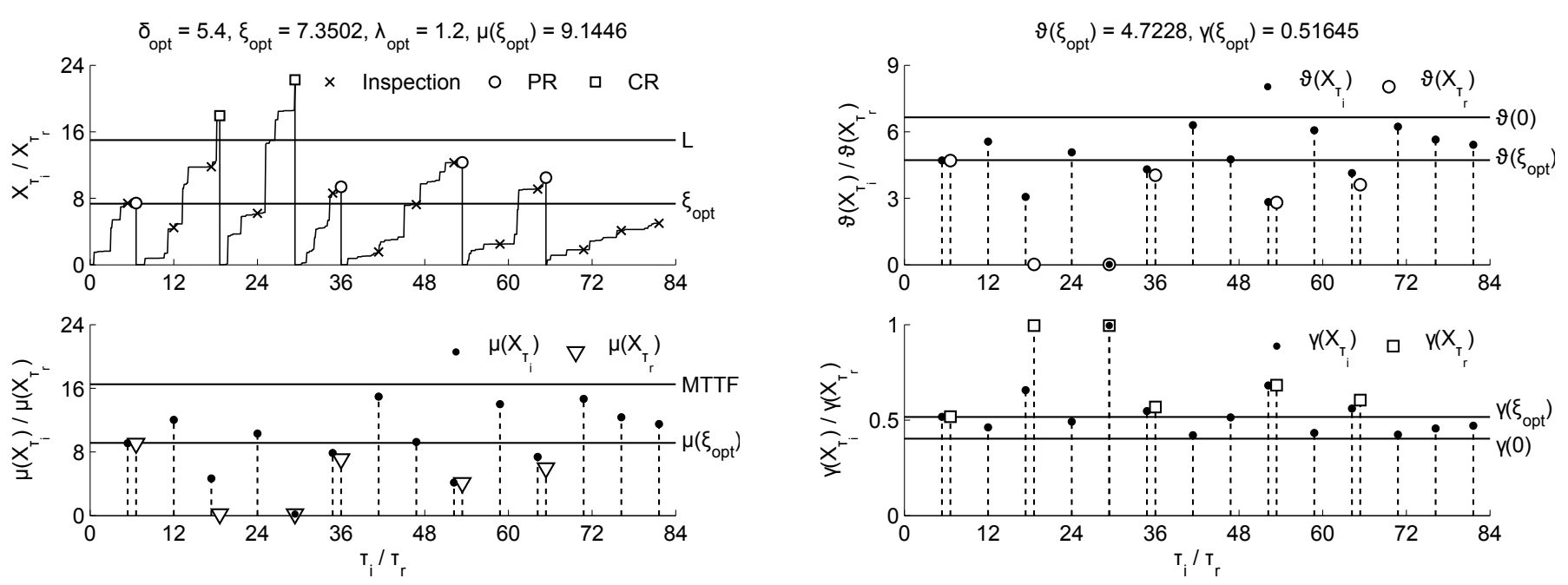

(a) Evolution of $X_{\tau_{i}} \mid X_{\tau_{r}}$ and $\mu\left(X_{\tau_{i}}\right) \mid \mu\left(X_{\tau_{r}}\right)$

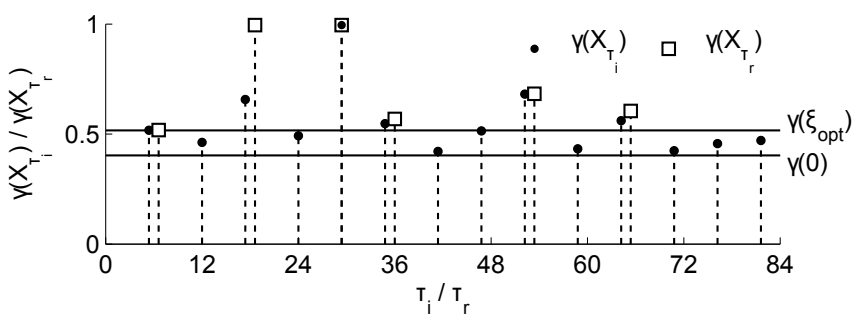

(b) Evolution of $\vartheta\left(X_{\tau_{i}}\right) \mid \vartheta\left(X_{\tau_{r}}\right)$ and $\gamma\left(X_{\tau_{i}}\right) \mid \gamma\left(X_{\tau_{r}}\right)$

Figure 5: Illustration of the $(\delta, \xi, \lambda)$ policy

2) $(\delta, \xi, \varphi)$ policy: The condition-based approach attempts to adapt the waiting time $\psi$ to the current or future system state. In this policy, $\psi$ is scheduled according to the conditional system reliability given the system deterioration level detected by the last inspection of a certain renewal cycle. Assuming that $\tau_{i}$ is this last inspection time and $X_{\tau_{i}}=y$, the reliability-based waiting time at $\tau_{i}$ is defined as

$$
\psi(y) \triangleq \sup \left\{u>0, R\left(\tau_{i}+u \mid y\right) \geq \varphi\right\},
$$

where $R\left(\tau_{i}+u \mid y\right)$ is given by (6) and $\varphi, 0 \leq \varphi \leq 1$, is a quantile level. Such a definition of $\psi$ does not only allows an adaptive waiting time, but it also guarantees that the reliability of the system, since the last inspection time $\tau_{i}$, is at least $\varphi$. Optimizing this maintenance policy returns to find the optimal values of the 3 decision variables: the inspection period $\delta$, the deterioration threshold to control prognosis precision $\xi$, and the quantile $\varphi$; thus it is called $(\delta, \xi, \varphi)$. Using the maintenance cost model in Section IV, and always for the same considered example system, the optimal configuration of the $(\delta, \xi, \varphi)$ policy is obtained at $\delta_{o p t}=6$, $\xi_{\text {opt }}=5.4028$ and $\varphi_{\text {opt }}=0.88$. We can see that $\xi_{\text {opt }}$ here is much smaller than the one of the optimal $(\delta, \xi, \lambda)$ policy, thus the reliability-based waiting time allows tuning the precision level of prognosis more softly than a constant waiting time. Furthermore, the optimal inspection period $\delta_{\text {opt }}$ of the $(\delta, \xi, \varphi)$ policy is longer than the one of the $(\delta, \xi, \lambda)$ policy. This leads to more saving in maintenance cost for the $(\delta, \xi, \varphi)$ policy. The behavior of the maintained system in the optimal configuration of the $(\delta, \xi, \varphi)$ policy is also shown in Figures $6 \mathrm{a}$ and $6 \mathrm{~b}$. Compared to the $(\delta, \xi, \lambda)$ policy, the waiting time in the $(\delta, \xi, \varphi)$ policy can be adapted now to the deterioration level at a last inspection time to trigger more appropriate replacement operations (see e.g., first replacement operations in Figures 5a and 6a).
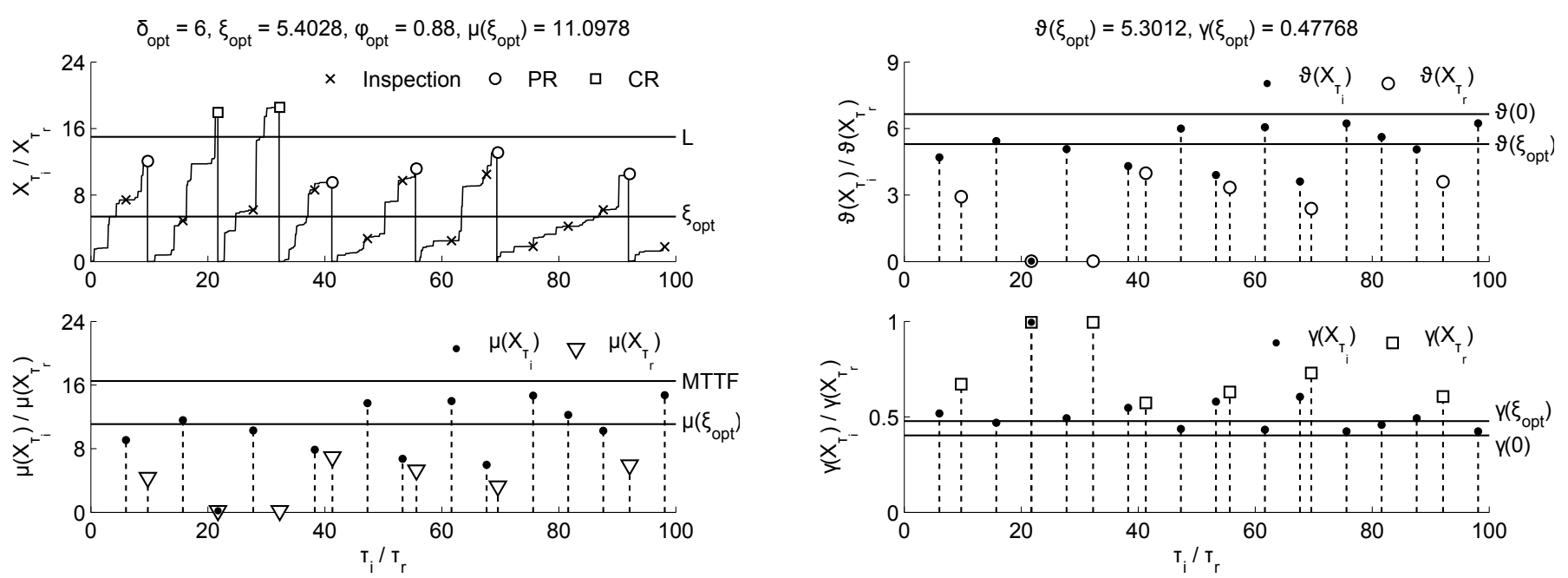

(a) Evolution of $X_{\tau_{i}} \mid X_{\tau_{r}}$ and $\mu\left(X_{\tau_{i}}\right) \mid \mu\left(X_{\tau_{r}}\right)$

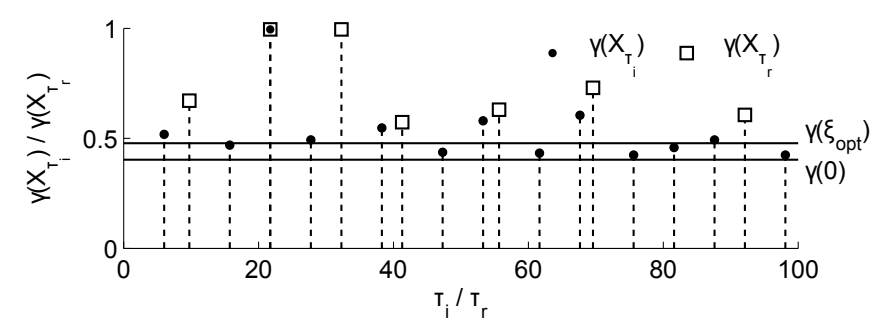

(b) Evolution of $\vartheta\left(X_{\tau_{i}}\right) \mid \vartheta\left(X_{\tau_{r}}\right)$ and $\gamma\left(X_{\tau_{i}}\right) \mid \gamma\left(X_{\tau_{r}}\right)$

Figure 6: Illustration of the $(\delta, \xi, \varphi)$ policy 
3) $(\delta, \xi, \eta)$ policy: Besides the performance, the robustness is another essential criterion in maintenance decision-making (review Section I for the definition of the performance and robustness of a maintenance policy). The constant waiting time irrespective of system state as in the $(\delta, \xi, \lambda)$ policy seems robust but less efficient, while the reliability-based waiting time as in the $(\delta, \xi, \varphi)$ policy allows a high performance but suffers loss in robustness. To achieve both performance and robustness for the maintenance policy, we define the waiting time from the last inspection $\tau_{i}$ of a given renewal cycle as follows

$$
\psi(y) \triangleq\left(\mu\left(\tau_{i} \mid y\right)-\eta\right) \cdot 1_{\left\{0 \leq \eta \leq \mu\left(\tau_{i} \mid y\right)\right\}},
$$

where $\mu\left(\tau_{i} \mid y\right)$ derived from (8) is the system conditional MRL at time $\tau_{i}$ given $X_{\tau_{i}}=y$, and $\eta$ is the associated safety time interval. The idea behind this choice is that $\mu\left(\tau_{i} \mid y\right)$ adaptive to the system state ensures the performance, whereas the constant $\eta$ to be optimized ensures the robustness. The maintenance policy admits the inspection period $\delta$, the deterioration threshold to control prognosis precision $\xi$ and the safety time interval $\eta$ as decision variables, so we call it $(\delta, \xi, \eta)$. With the same system and maintenance costs as in the previous illustrations, the $(\delta, \xi, \eta)$ policy reaches its optimal configuration at $\delta_{\text {opt }}=6$, $\xi_{\text {opt }}=5.5526$ and $\eta_{o p t}=4.8$. The similar values of $\delta_{o p t}$ and $\xi_{o p t}$ in both the $(\delta, \xi, \eta)$ policy and the $(\delta, \xi, \varphi)$ policy indicate that they both exploit equally well the information on the prognosis precision for maintenance decision-making; but we will see in Section V-B that the $(\delta, \xi, \eta)$ policy is more robust. An illustration of the optimal $(\delta, \xi, \eta)$ policy is represented in Figures $7 \mathrm{a}$ and $7 \mathrm{~b}$. We can remark that,
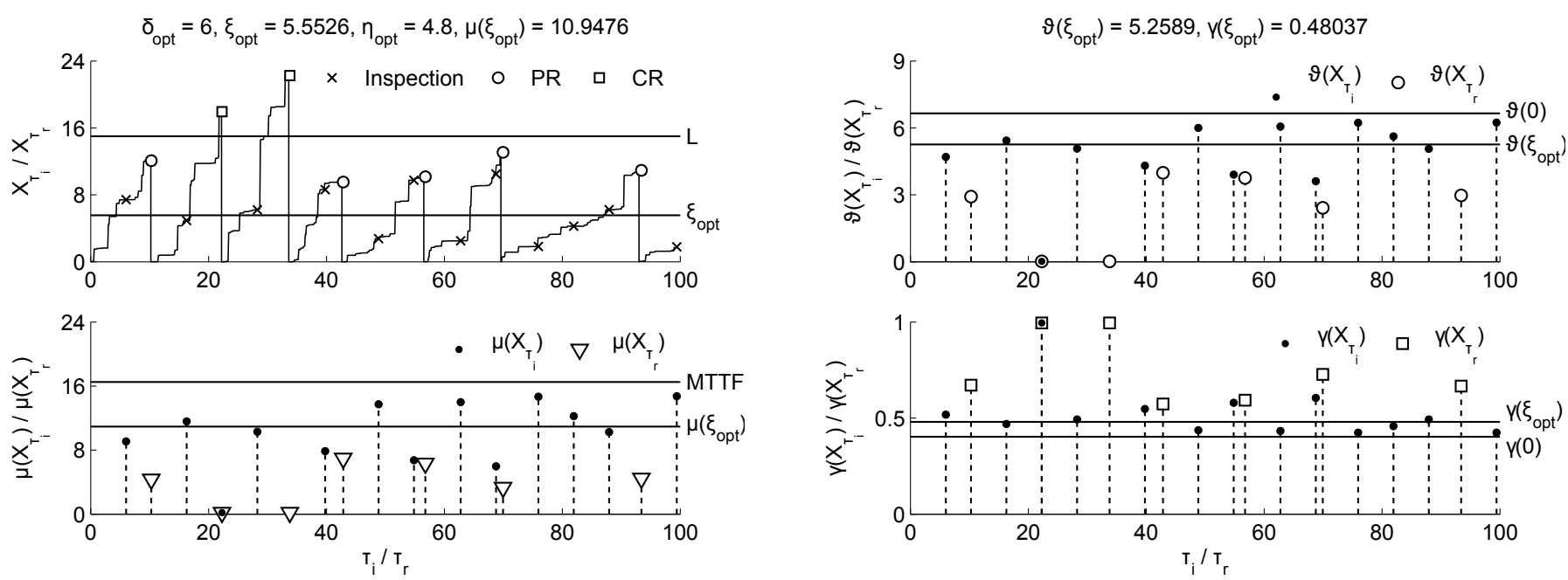

(a) Evolution of $X_{\tau_{i}} \mid X_{\tau_{r}}$ and $\mu\left(X_{\tau_{i}}\right) \mid \mu\left(X_{\tau_{r}}\right)$

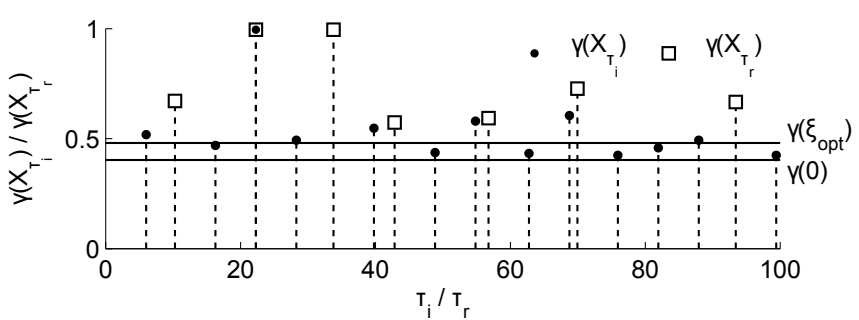

(b) Evolution of $\vartheta\left(X_{\tau_{i}}\right) \mid \vartheta\left(X_{\tau_{r}}\right)$ and $\gamma\left(X_{\tau_{i}}\right) \mid \gamma\left(X_{\tau_{r}}\right)$

Figure 7: Illustration of the $(\delta, \xi, \eta)$ policy

under their optimal configuration, the behaviors of the system maintained by the $(\delta, \xi, \eta)$ policy and the $(\delta, \xi, \varphi)$ policy are almost similar.

\section{Maintenance Cost Model, And Optimization}

This section aims at building a mathematical cost model to evaluate $C_{\infty}$ in (13) in order to assess the performance of the new predictive maintenance decision framework. In many published works, the cost rate $C_{\infty}$ is evaluated analytically resorting to the renewal-reward theorem [84]. This classical method seems useful for static maintenance decision rules [40]. When the decision rules are dynamic, it is more interesting to take advantage of the semi-regenerative theory [59, chapter 10]. Indeed, under the assumptions of Markovian deterioration process and of the considered maintenance framework, an intervention (i.e., either an inspection or a replacement) decision depends only on the measured deterioration level at an inspection time, and an intervention merely adjusts the system deterioration level without modifying the underlying deterioration mechanism. Consequently, the future evolution of the maintained system after an inspection depends only on the deterioration level revealed at this time. As such, let $\tilde{X}_{t}$ be a scalar random variable characterizing the state of the maintained system at time $t$, and $\tau_{i}$ is the $i$-th inspection time, the processes $\left\{\tilde{X}_{\tau_{i}+t}\right\}_{t \geq 0}$ conditioned on $\tilde{X}_{\tau_{i}}$ and $\left\{\tilde{X}_{t}\right\}_{t>0}$ conditioned on $\tilde{X}_{0}$ follow the same probability law. This property implies that $\left\{\tilde{X}_{t}\right\}_{t>0}$ is a semi-regenerative process with semi-regeneration times $\tau_{i}, i=1,2, \ldots$ [59, page 356], and that the time interval between successive inspection times is a semi-regenerative cycle. Embedded in such a semi-regenerative process, there is an Markov chain $\left\{Y_{i}\right\}_{i \in \mathbb{N}}$, $Y_{i} \triangleq \tilde{X}_{\tau_{i}}$, with continuous state space $[0, \infty[$ and stationary law $\pi$, describing the maintained system behavior at inspection times. Thus, the semi-regenerative cycle is also known as a Markov renewal cycle. The study of the asymptotic behavior of $\left\{\tilde{X}_{t}\right\}_{t \geq 0}$ can 
be restricted to a Markov renewal cycle, accordingly we can compute the long-run expected maintenance cost rate in (13) as (see e.g. [20] for the detailed proof)

$$
\begin{aligned}
C_{\infty}=\frac{E_{\pi}\left[C\left(\left[\tau_{i-1}^{-}, \tau_{i}^{-}\right]\right)\right]}{E_{\pi}[\Delta \tau]}=C_{i} \cdot \frac{E_{\pi}\left[N_{i}\left(\left[\tau_{i-1}^{-}, \tau_{i}^{-}\right]\right)\right]}{E_{\pi}[\Delta \tau]}+C_{p} \cdot \frac{E_{\pi}\left[N_{p}\left(\left[\tau_{i-1}^{-}, \tau_{i}^{-}\right]\right)\right]}{E_{\pi}[\Delta \tau]} & \\
& +C_{c} \cdot \frac{E_{\pi}\left[N_{c}\left(\left[\tau_{i-1}^{-}, \tau_{i}^{-}\right]\right)\right]}{E_{\pi}[\Delta \tau]}+C_{d} \cdot \frac{E_{\pi}\left[W\left(\left[\tau_{i-1}^{-}, \tau_{i}^{-}\right]\right)\right]}{E_{\pi}[\Delta \tau]}
\end{aligned}
$$

where $\left[\tau_{i-1}^{-}, \tau_{i}^{-}\right], i=1,2, \ldots$, is a single Markov renewal cycle, $\Delta \tau=\tau_{i}^{-}-\tau_{i-1}^{-}$, and $E_{\pi}$ denotes the expectation with respect to $\pi$. In the following, we focus on the formulation of the stationary law $\pi$ and of the expectation quantities in (17).

\section{A. Stationary Law of the Maintained System State}

As aforementioned, we can characterize the behavior of maintained system at inspection times by the stationary law $\pi$ of the Markov chain with continuous state space $\left[0, \infty\left[,\left\{Y_{i}\right\}_{i \in \mathbb{N}^{N}}\right.\right.$ Let consider the Markov renewal cycle $\left[\tau_{i-1}^{-}, \tau_{i}^{-}\right], i=1,2, \ldots, y$ and let $x$ be the deterioration levels of maintained system at the beginning and the end of the cycle respectively (i.e., $X_{\tau_{i-1}^{-}}=y$ and $X_{\tau_{i}^{-}}=x$ ), then the stationary law $\pi$ is solution of the invariance equation:

$$
\pi(x)=\int_{0}^{\infty} F(x \mid y) \pi(y) d y
$$

where $F(x \mid y)$ is the deterioration transition law from $y$ to $x$, that can be derived from an exhaustive analysis of all the possible evolution and maintenance scenarios on the Markov renewal cycle $\left[\tau_{i-1}^{-}, \tau_{i}^{-}\right]$:

- Scenario 1: $y<\xi$. There is no change in the system state at $\tau_{i-1}^{+}$(i.e., $X_{\tau_{i-1}^{+}}=X_{\tau_{i-1}^{-}}=y$ ) and the next inspection is scheduled $\delta$ time units later (i.e., at $\tau_{i}=\tau_{i-1}+\delta$ ). The deterioration increment from $\tau_{i-1}^{+}$to $\tau_{i}^{-}, X_{\tau_{i}^{-}}-X_{\tau_{i-1}^{+}}$, follows a probability density $f_{\alpha \delta, \beta}(x-y)$.

- Scenario 2: $\xi \leq y<L$. There is no change in the system state at $\tau_{i-1}^{+}$(i.e., $X_{\tau_{i-1}^{+}}=X_{\tau_{i-1}^{-}}=y$ ) and the next inspection is scheduled $\psi(y)+\delta$ time units later (i.e., at $\tau_{i}=\tau_{i-1}+\psi(y)+\delta$ ). On the time interval $\left[\tau_{i-1}^{+}, \tau_{i}^{-}\right]$, the system is replaced once (either preventively or correctively) at time $\tau_{r}=\tau_{i-1}+\psi(y)$. After the instantaneous replacement with negligible time, the system is as good as new (i.e., $X_{\tau_{r}^{+}}=0$ ). Assuming $X_{\tau_{r}^{-}}=z, z \geq y$, the deterioration increment from $\tau_{i-1}^{+}$to $\tau_{r}^{-}$, $X_{\tau_{r}^{-}}-X_{\tau_{i-1}^{+}}$, follows a probability density $f_{\alpha \psi(y), \beta}(z-y)$, while the deterioration increment from $\tau_{r}^{+}$to $\tau_{i}^{-}, X_{\tau_{i}^{-}}-X_{\tau_{r}^{+}}$, follows a probability density $f_{\alpha \delta, \beta}(x)$. Both increments $X_{\tau_{r}^{-}}-X_{\tau_{i-1}^{+}}$and $X_{\tau_{i}^{-}}-X_{\tau_{r}^{+}}$are independent, the probability law of the deterioration "increment" from $\tau_{i-1}^{+}$to $\tau_{i}^{-}, X_{\tau_{i}^{-}}-X_{\tau_{i-1}^{+}}$, is thus given by $f_{\alpha \delta, \beta}(x) \cdot \int_{y}^{\infty} f_{\alpha \psi(y), \beta}(z-y) d z$.

- Scenario 3: $y \geq L$. The system is correctively replaced at time $\tau_{i-1}$ and the next inspection is scheduled $\delta$ time units later (i.e., at $\tau_{i}=\tau_{i-1}+\delta$ ). After the instantaneous replacement with negligible time, the system is as good as new (i.e., $X_{\tau_{i-1}^{+}}=0$ ). As such, the deterioration increment from $\tau_{i-1}^{+}$to $\tau_{i}^{-}, X_{\tau_{i}^{-}}-X_{\tau_{i-1}^{+}}$, follows a probability density $f_{\alpha \delta, \beta}(x)$.

In the above scenarios, $f_{\alpha \psi(y), \beta}(\cdot)$ and $f_{\alpha \delta, \beta}(\cdot)$ are given from (1). Thus, the deterioration transition law $F(x \mid y)$ is obtained as

$$
F(x \mid y)=f_{\alpha \delta, \beta}(x-y) \cdot 1_{\{y<\xi\}}+f_{\alpha \delta, \beta}(x) \cdot \int_{y}^{\infty} f_{\alpha \psi(y), \beta}(z-y) d z \cdot 1_{\{\xi \leq y<L\}}+f_{\alpha \delta, \beta}(x) \cdot 1_{\{y \geq L\}} .
$$

From (18) and (19), the invariant equation of the stationary law $\pi$ is rewritten as

$$
\pi(x)=\int_{0}^{\xi} f_{\alpha \delta, \beta}(x-y) \pi(y) d y+f_{\alpha \delta, \beta}(x) \cdot \int_{\xi}^{L}\left(\int_{y}^{\infty} f_{\alpha \psi(y), \beta}(z-y) d z\right) \pi(y) d y+f_{\alpha \delta, \beta}(x) \cdot \int_{L}^{\infty} \pi(y) d y .
$$

Mathematically, (20) has the form of a homogeneous integral equation of the second kind, and we have to solve it to obtain the stationary law $\pi(x)$. In the literature, many analytic and numerical solution methods have been proposed to solve such an integral equation (see e.g., [85]). Here, we remark that (20) can be represented as $\pi(x)=g(\pi(x))$ where $g(\cdot)$ is a continuous function, $\pi(x)$ is then a fixed point of $g$. So, we can numerically evaluate $\pi(x)$ by adapting fixed-point iteration algorithm method for (20). By this way, we approximate (20) by the following iterated function

$$
\omega_{k}(x)=\int_{0}^{\xi} f_{\alpha \delta, \beta}(x-y) \omega_{k-1}(y) d y+f_{\alpha \delta, \beta}(x) \cdot \int_{\xi}^{L}\left(\int_{y}^{\infty} f_{\alpha \psi(y), \beta}(z-y) d z\right) \omega_{k-1}(y) d y+f_{\alpha \delta, \beta}(x) \cdot \int_{L}^{\infty} \omega_{k-1}(y) d y,
$$

where $\omega_{k}(x), k=1,2, \ldots$, is the quantity obtained at $k$-th iteration (we can set its initial value $\left.\omega_{1}(x)=1\right)$, and $\pi(x)=$ $\lim _{k \rightarrow \infty} \omega_{k}(x)$. Many numerical tests have shown that the $\omega_{k}(x)$ converges very quickly to the true stationary law $\pi(x)$ (see also Section IV-C for illustrations of the quick convergence of the proposed numerical method). 


\section{B. Expected Quantities With Respect to the Stationary Law}

1) Expected length of a Markov renewal cycle: Let consider the Markov renewal cycle $\left[\tau_{i-1}^{-}, \tau_{i}^{-}\right], i=1,2, \ldots$, where $\tau_{i}^{-}$denotes the times just before the $i$-th inspection, its length $\Delta \tau=\tau_{i}^{-}-\tau_{i-1}^{-}$closely depends on the system state revealed at the beginning of the cycle (i.e., on $X_{\tau_{i-1}^{-}}=y$ ). Indeed, under the proposed predictive maintenance decision framework, the next inspection will be either at $\tau_{i}=\tau_{i-1}+\delta$ if $y<\xi$ or $y \geq L$, or at $\tau_{i}=\tau_{i-1}+\psi(y)+\delta$ otherwise (i.e., $\xi \leq y<L$ ). Thus, we can express the length of the Markov renewal cycle as

$$
\Delta \tau=\tau_{i}^{-}-\tau_{i-1}^{-}=\delta \cdot 1_{\{y<\xi\}}+\delta \cdot 1_{\{y \geq L\}}+(\psi(y)+\delta) \cdot 1_{\{\xi \leq y<L\}}=\delta+\psi(y) \cdot 1_{\{\xi \leq y<L\}} \cdot
$$

Its expected value with respect to the stationary law $\pi$ is then

$$
E_{\pi}[\Delta \tau]=E_{\pi}\left[\delta+\psi(y) \cdot 1_{\{\xi \leq y<L\}}\right]=\delta+\int_{\xi}^{L} \psi(y) \pi(y) d y .
$$

2) Expected number of inspections on a Markov renewal cycle: On a Markov renewal cycle, one and only one inspection is performed. As such, the associated expected number of inspections with respect to the stationary law $\pi$ is always equal to 1

$$
E_{\pi}\left[N_{i}\left(\left[\tau_{i-1}^{-}, \tau_{i}^{-}\right]\right)\right]=1
$$

3) Expected number of PR on a Markov renewal cycle: Associated with PR operations, the Markov renewal cycle is $\left[\tau_{i-1}^{-}, \tau_{i}^{-}\right]$, in which $\tau_{i}=\tau_{i-1}+\psi(y)+\delta$. On this cycle, the running system is preventively replaced only once at $\tau_{r}=\tau_{i-1}+\psi(y)$ if and only if $X_{\tau_{i-1}^{-}}=y$ and $\xi \leq X_{\tau_{i-1}^{-}}<X_{\tau_{r}^{-}}<L$. Thus, the associated expected number of PR operations with respect to the stationary law $\pi$ can be computed as

$$
\left.E_{\pi}\left[N_{p}\left(\left[\tau_{i-1}^{-}, \tau_{i}^{-}\right]\right)\right]=E_{\pi}\left[1_{\left\{\xi \leq X_{\tau_{i-1}^{-}}<X_{\tau_{r}^{-}}<L, X_{\tau_{i-1}^{-}}\right.}=y\right\}\right]=\int_{\xi}^{L} F_{\alpha \psi(y), \beta}(L-y) \pi(y) d y,
$$

where $F_{\alpha \psi(y), \beta}(\cdot)$ is derived from (2).

4) Expected number of CR on a Markov renewal cycle: CR operations can be carried out once or twice on the Markov renewal cycle $\left[\tau_{i-1}^{-}, \tau_{i}^{-}\right]$depending on the value of $X_{\tau_{i-1}^{-}}$. When $X_{\tau_{i-1}^{-}}=y<\xi$, the failed system can be correctively replaced only once at $\tau_{i}=\tau_{i-1}+\delta$ if it fails between $\tau_{i-1}^{-}$and $\tau_{i}^{-}$(i.e., $X_{\tau_{i-1}^{-}}<\xi<L \leq X_{\tau_{i}^{-}}$). We can therefore compute the expected number of CR operations performed on $\left[\tau_{i-1}^{-}, \tau_{i}^{-}\right]$, where $\tau_{i}=\tau_{i-1}+\delta$, with respect to the stationary law $\pi$ as

$$
E_{\pi}\left[1_{\left\{X_{\tau_{i-1}^{-}}<\xi<L \leq X_{\tau_{i}^{-}}, X_{\tau_{i-1}^{-}}=y\right\}}\right]=\int_{0}^{\xi} \bar{F}_{\alpha \delta, \beta}(L-y) \pi(y) d y .
$$

When $\xi \leq X_{\tau_{i-1}^{-}}=y<L$, the Markov renewal cycle becomes $\left[\tau_{i-1}^{-}, \tau_{i}^{-}\right]$, where $\tau_{i}=\tau_{i-1}+\psi(y)+\delta$. On this cycle, the failed system can be correctively replaced one or twice at $\tau_{r}=\tau_{i-1}+\psi(y)$ if it fails between $\tau_{i-1}^{-}$and $\tau_{r}^{-}$(i.e., $X_{\tau_{i-1}^{-}}<L \leq X_{\tau_{r}^{-}}$), and/or at $\tau_{i}=\tau_{i-1}+\psi(y)+\delta$ if the system fails between $\tau_{r}^{+}$and $\tau_{i}^{-}$(i.e., $0=X_{\tau_{r}^{+}}<L \leq X_{\tau_{i}^{-}}$). As such, the expected number of CR operations performed on $\left[\tau_{i-1}^{-}, \tau_{i}^{-}\right]$, where $\tau_{i}=\tau_{i-1}+\psi(y)+\delta$, with respect to the stationary law $\pi$ can by computed as

$$
\begin{array}{r}
\left.E_{\pi}\left[1_{\left\{\xi \leq X_{\tau_{i-1}^{-}}<L \leq X_{\tau_{r}^{-}}, X_{\tau_{i-1}^{-}}\right.}=y\right\}\right]+E_{\pi}\left[1_{\left.\left\{\xi \leq X_{\tau_{i-1}^{-}}<L, X_{\tau_{r}^{+}}<L \leq X_{\tau_{i}^{-}}, X_{\tau_{r}^{+}}=0, X_{\tau_{i-1}^{-}}=y\right\}\right]}=\int_{\xi}^{L} \bar{F}_{\alpha \psi(y), \beta}(L-y) \pi(y) d y+\bar{F}_{\alpha \delta, \beta}(L) \int_{\xi}^{L} \pi(y) d y .\right.
\end{array}
$$

Consequently, the associated expected number of CR operations with respect to the stationary law $\pi$ is given by

$$
E_{\pi}\left[N_{c}\left(\left[\tau_{i-1}^{-}, \tau_{i}^{-}\right]\right)\right]=\int_{0}^{\xi} \bar{F}_{\alpha \delta, \beta}(L-y) \pi(y) d y+\int_{\xi}^{L} \bar{F}_{\alpha \psi(y), \beta}(L-y) \pi(y) d y+\bar{F}_{\alpha \delta, \beta}(L) \int_{\xi}^{L} \pi(y) d y,
$$

where $\bar{F}_{\alpha \delta, \beta}(\cdot)=1-F_{\alpha \delta, \beta}(\cdot)$ and $\bar{F}_{\alpha \psi(y), \beta}(\cdot)=1-F_{\alpha \psi(y), \beta}(\cdot), F_{\alpha \delta, \beta}(\cdot)$ and $F_{\alpha \psi(y), \beta}(\cdot)$ are given from (2).

5) Expected downtime on a Markov renewal cycle: Similarly, the expected downtime of the maintained system on a Markov renewal cycle with respect to the stationary law $\pi$ can be analyzed as follows. When the semi-renewal cycle is $\left[\tau_{i-1}^{-}, \tau_{i}^{-}\right]$, where $\tau_{i}=\tau_{i-1}+\delta$, the expected downtime with respect to the stationary law $\pi$ is

$$
E_{\pi}\left[\int_{\tau_{i-1}}^{\tau_{i}} 1_{\left\{X_{\tau_{i-1}^{-}}<\xi<L \leq X_{t}, X_{\tau_{i-1}^{-}}=y\right\}} d t\right]=\int_{0}^{\xi}\left(\int_{0}^{\delta} \bar{F}_{\alpha u, \beta}(L-y) d u\right) \pi(y) d y .
$$


When the semi-renewal cycle is $\left[\tau_{i-1}^{-}, \tau_{i}^{-}\right] \equiv\left[\tau_{i-1}^{-}, \tau_{r}^{-}\right] \cup\left[\tau_{r}^{+}, \tau_{i}^{-}\right]$, where $\tau_{r}=\tau_{i-1}+\psi(y)$ and $\tau_{i}=\tau_{i-1}+\psi(y)+\delta$, the expected downtime with respect to the stationary law $\pi$ becomes

$$
\begin{aligned}
E_{\pi}\left[\int_{\tau_{i-1}}^{\tau_{r}} 1_{\left\{\xi \leq X_{\tau_{i-1}^{-}}<L \leq X_{t}, X_{\tau_{i-1}^{-}}=y\right\}} d t\right]+E_{0} & {\left[\int_{\tau_{r}}^{\tau_{i}} 1_{\left\{\leq X_{\tau_{i-1}^{-}}<L, X_{\tau_{r}^{+}}<L \leq X_{t}, X_{\tau_{r}^{+}}=0, X_{\tau_{i-1}^{-}}=y\right\}} d t\right] } \\
& =\int_{\xi}^{L}\left(\int_{0}^{\psi(y)} \bar{F}_{\alpha u, \beta}(L-y) d u\right) \pi(y) d y+\int_{\xi}^{L} \pi(y) d y \cdot \int_{0}^{\delta} \bar{F}_{\alpha u, \beta}(L) d u .
\end{aligned}
$$

As a result, one can obtain the associated expected system downtime of the system with respect to the stationary law $\pi$ as

$$
\begin{aligned}
E_{\pi}\left[W\left(\left[\tau_{i-1}^{-}, \tau_{i}^{-}\right]\right)\right]= & \int_{0}^{\xi}\left(\int_{0}^{\delta} \bar{F}_{\alpha u, \beta}(L-y) d u\right) \pi(y) d y \\
& +\int_{\xi}^{L}\left(\int_{0}^{\psi(y)} \bar{F}_{\alpha u, \beta}(L-y) d u\right) \pi(y) d y+\int_{\xi}^{L} \pi(y) d y \cdot \int_{0}^{\delta} \bar{F}_{\alpha u, \beta}(L) d u,
\end{aligned}
$$

where $\bar{F}_{\alpha u, \beta}(\cdot)=1-F_{\alpha u, \beta}(\cdot)$, and $F_{\alpha u, \beta}(\cdot)$ is given by (2).

\section{Maintenance Policies Optimization}

Using (21), and introducing (23), (24), (25), (26) and (27) into (17), we obtain the full mathematical cost model of the proposed predictive maintenance decision framework. The traditional framework is a particular case of the new one, so we can derive its cost model from (17) by taking $\psi=0$. In the following, we show the existence of the optimal configuration of each proposed maintenance policy by numerical experiments. The experiments are based on the same system characteristics and on the same set of maintenance costs as the ones used in Section III. The shapes of the stationary law and the maintenance cost rate of the $(\delta, \xi, \lambda)$ policy, the $(\delta, \xi, \varphi)$ policy and the $(\delta, \xi, \eta)$ policy are illustrated in Figures 8, 9 and 10. These results are given by numerical computation of (17) and (21), and are verified by Monte Carlo simulation. For the system maintained by the $(\delta, \xi, \lambda)$ policy, the evolution of numerical evaluation of the stationary law (i.e., $\omega_{k}(x)$ ) with respect to the iteration number $k$ is shown on the left of Figure 8 a. The convergence of $\omega_{k}(x)$ towards $\pi(x)$ is very fast (i.e., from $k=4$ ), hence a good performance of the proposed iteration algorithm (see the end of Section IV-A). The 2 almost identical curves of $\pi(x)$ obtained by numerical computation according to (21) and by kernel density estimation method [86] on the right of Figure 8a justify the exactness of the mathematical formulation. Figures $8 \mathrm{~b}$, $8 \mathrm{c}$ and $8 \mathrm{~d}$ represent the long-run expected maintenance cost rate of the $(\delta, \xi, \lambda)$ policy when one of the decision variables is fixed at its optimal value and the others vary. The form of the cost rate surface justifies the existence of a global minimum, and hence finding optimal decision variables is needed to obtain the best performance of the maintenance policy. Figures 9 and 10 have to be read in the same way as Figure 8 . Given the cost model, optimizing the policies $(\delta, \xi, \lambda),(\delta, \xi, \varphi)$ and $(\delta, \xi, \eta)$ returns to find the set of decision variables of each policy that minimizes the associated long-run expected cost maintenance rate

$$
\begin{aligned}
& C_{\infty}\left(\delta_{o p t}, \xi_{o p t}, \lambda_{o p t}\right)=\min _{(\delta, \xi, \lambda)}\left\{C_{\infty}(\delta, \xi, \lambda), \delta \geq 0,0 \leq \xi \leq L, \lambda \geq 0\right\}, \\
& C_{\infty}\left(\delta_{o p t}, \xi_{o p t}, \varphi_{o p t}\right)=\min _{(\delta, \xi, \varphi)}\left\{C_{\infty}(\delta, \xi, \varphi), \delta \geq 0,0 \leq \xi \leq L, 0 \leq \varphi \leq 1\right\}, \\
& C_{\infty}\left(\delta_{o p t}, \xi_{o p t}, \eta_{o p t}\right)=\min _{(\delta, \xi, \eta)}\left\{C_{\infty}(\delta, \xi, \eta), \delta \geq 0,0 \leq \xi \leq L, \eta \geq 0\right\} .
\end{aligned}
$$

In the present paper, we apply the generalized pattern search algorithm, a derivative free algorithm for black-box optimization, presented in $[87,88]$ to find the optimal maintenance cost rate and the associated optimal decision variables. The patternsearch solver of Matlab's Global Optimization Toolbox has been used. Many numerical experiments show that the convergence to the optimal solution is very quick (about couple of seconds) for whatever initial points and for a very high precision $\left(10^{-10}\right)$. Table I shows the optimal tuning for each of the considered policies. Among the considered policies, the optimal maintenance cost rates of

Table I: Optimal configuration of the $(\delta, \xi, \lambda)$ policy, the $(\delta, \xi, \varphi)$ policy and the $(\delta, \xi, \eta)$ policy

\begin{tabular}{ccc}
\hline Policy & Optimal decision variables & Optimal cost rate \\
\hline$(\delta, \xi, \lambda)$ & $\delta_{\text {opt }}=5.4, \xi_{\text {opt }}=7.3502, \lambda_{\text {opt }}=1.2$ & $C_{\infty}\left(\delta_{o p t}, \xi_{\text {opt }}, \lambda_{\text {opt }}\right)=6.2842$ \\
$(\delta, \xi, \varphi)$ & $\delta_{\text {opt }}=6, \xi_{\text {opt }}=5.4028, \varphi_{\text {opt }}=0.88$ & $C_{\infty}\left(\delta_{o p t}, \xi_{\text {opt }}, \varphi_{\text {opt }}\right)=5.9857$ \\
$(\delta, \xi, \eta)$ & $\delta_{\text {opt }}=6, \xi_{\text {opt }}=5.5526, \eta_{\text {opt }}=4.8$ & $C_{\infty}\left(\delta_{\text {opt }}, \xi_{\text {opt }}, \eta_{\text {opt }}\right)=5.9746$ \\
\hline
\end{tabular}

the $(\delta, \xi, \lambda)$ policy is the most important, so it is the least profitable policy. The $(\delta, \xi, \varphi)$ policy and the $(\delta, \xi, \eta)$ policy share almost 

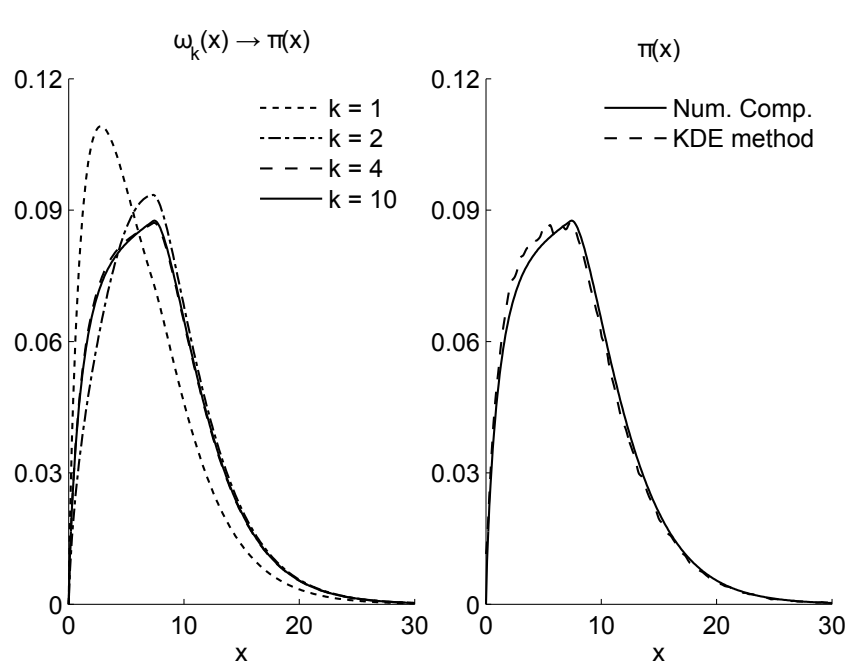

(a) $\omega_{k}(x)$ and $\pi(x)$

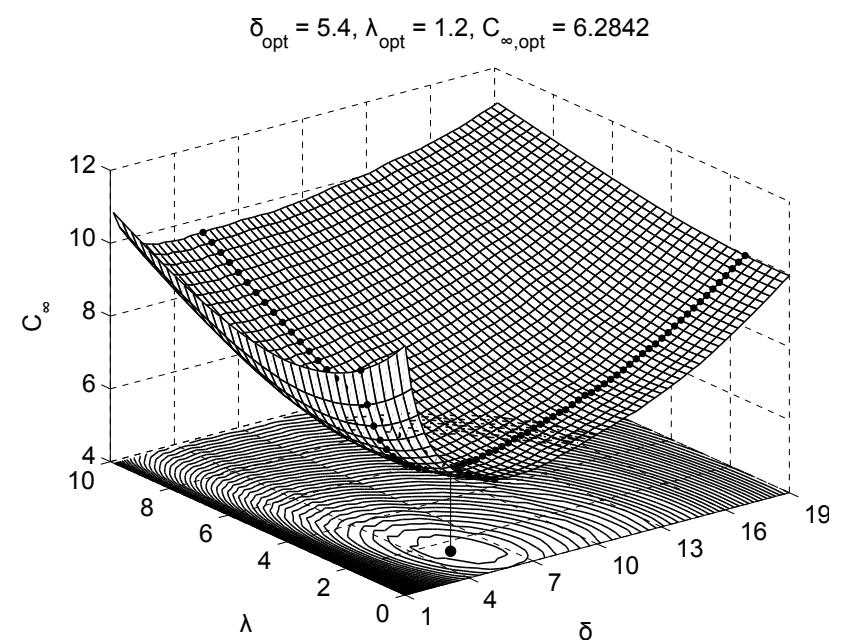

(c) $C_{\infty}\left(\delta, \xi_{\text {opt }}, \lambda\right)$

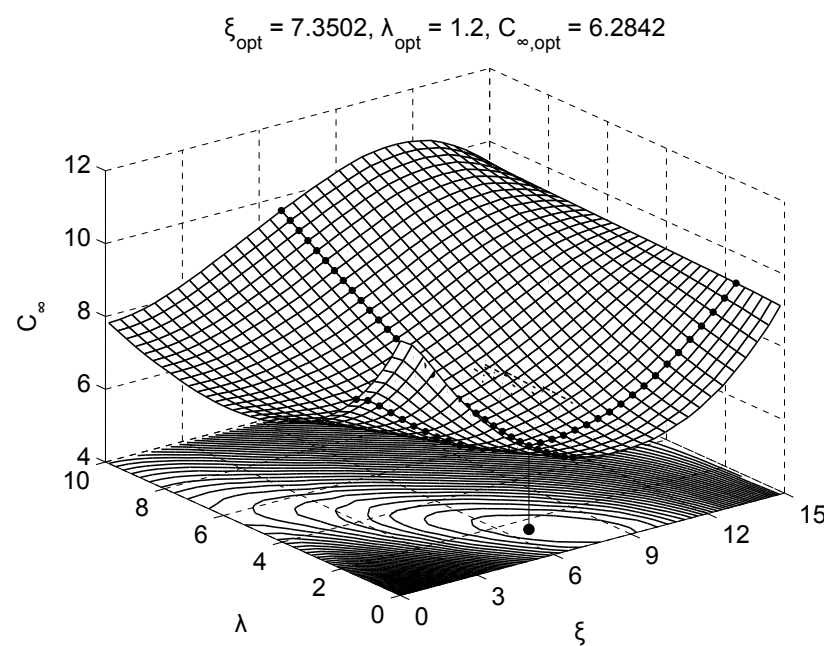

(b) $C_{\infty}\left(\delta_{o p t}, \xi, \lambda\right)$

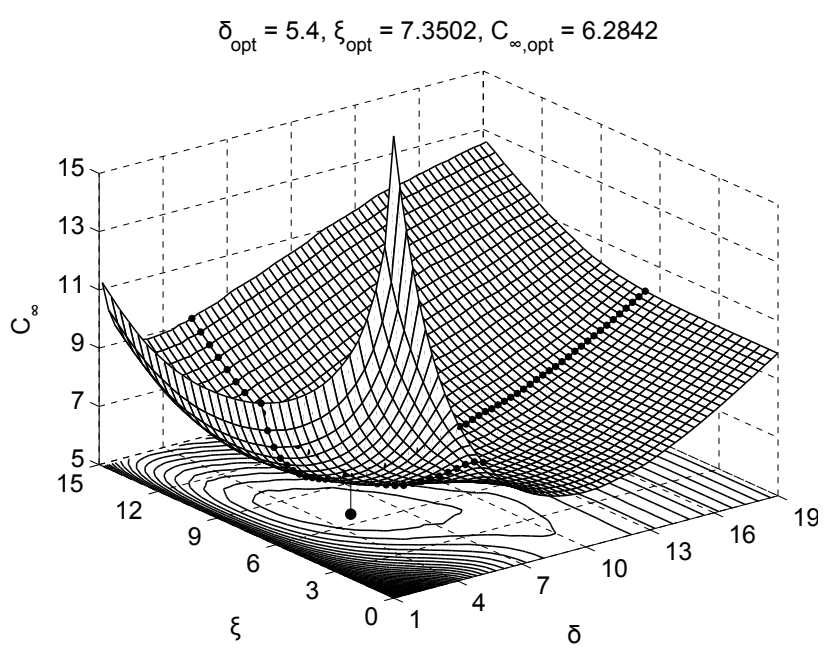

(d) $C_{\infty}\left(\delta, \xi, \lambda_{o p t}\right)$

Figure 8: Illustration of the stationary law and the cost rate of the system maintained under the $(\delta, \xi, \lambda)$ policy

identical cost rates, they have thus the same maintenance effectiveness. Still, these remarks only stem from observations made on the considered example system. To have more general conclusions and to gain a better insight into the behavior of the proposed policies, further studies on their performance and robustness are conducted in Section V.

\section{Performance and Robustness Assessment of the Proposed Predictive Maintenance Decision Framework}

This section aims at seeking more general conclusions on the effectiveness of the proposed predictive maintenance decision framework. To this end, we compare the performance of the $(\delta, \xi, \lambda)$ policy, the $(\delta, \xi, \varphi)$ policy and the $(\delta, \xi, \eta)$ policy to the $(\delta, \zeta)$ policy, and analyze their robustness under various configurations of maintenance operations costs and system characteristics. Recall that the performance of a maintenance policy is defined as its capacity to save maintenance costs under its optimal configuration, and its robustness is seen as its ability to keep its cost saving close to the optimum when it is out of its optimal configuration. We propose then to use the so-called relative gain in the optimal long-run expected maintenance cost rate [24] to assess the maintenance performance of a certain policy $A$ compared with a certain policy $B$

$$
\kappa_{C}(\%)=\frac{C_{\infty, o p t}^{B}-C_{\infty, o p t}^{A}}{C_{\infty, \text { opt }}^{B}} \times 100 \%,
$$

where $C_{\infty, \text { opt }}^{A}$ and $C_{\infty, \text { opt }}^{B}$ are the optimal long-run expected maintenance cost rate of the policies $A$ and $B$. If $\kappa_{C}(\%)>0$, the policy $A$ is more profitable than the policy $B$; if $\kappa_{C}(\%)=0$, they have the same profit; and finally if $\kappa_{C}(\%)<0$, the policy $A$ is less profitable than the policy $B$. To assess the robutness of a maintenance policy, we resort to the so-called relative increase in its long-run expected maintenance cost rate defined as [89]

$$
\epsilon_{C}(\%)=\frac{C_{\infty}-C_{\infty, o p t}}{C_{\infty, o p t}} \times 100 \%,
$$



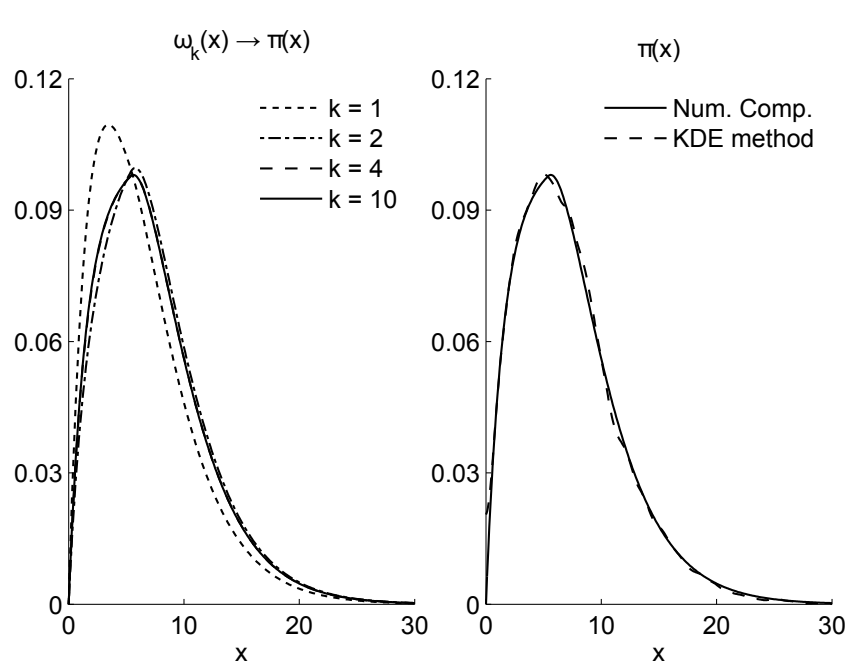

(a) $\omega_{k}(x)$ and $\pi(x)$

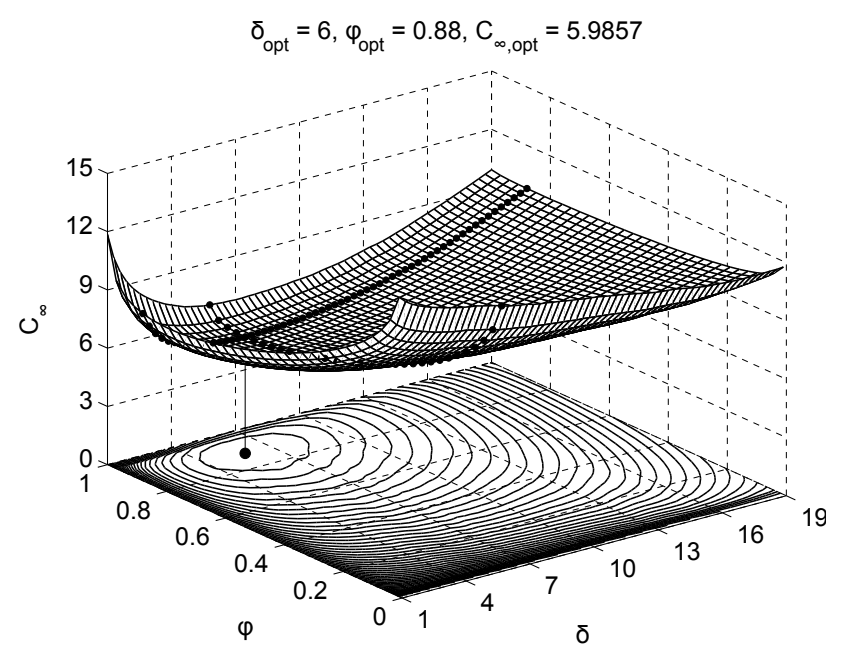

(c) $C_{\infty}\left(\delta, \xi_{\text {opt }}, \varphi\right)$

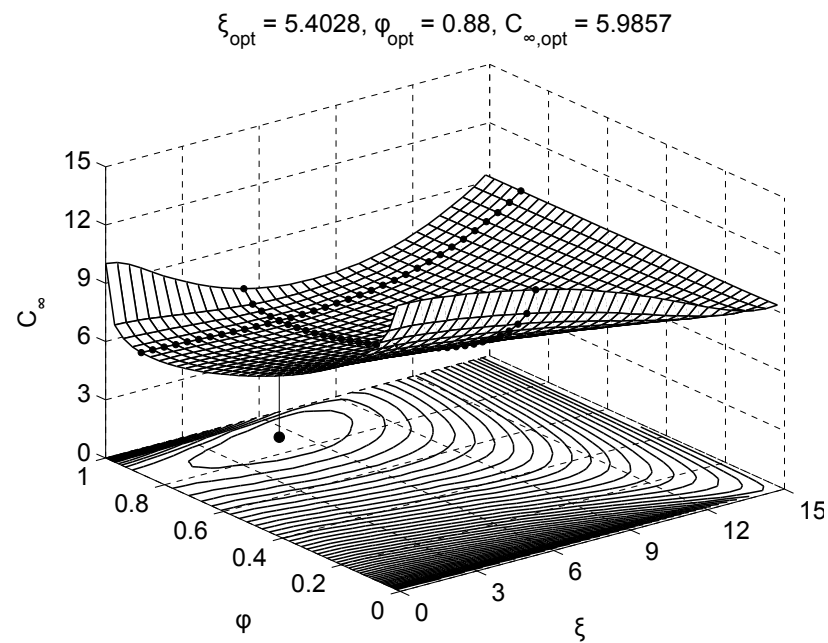

(b) $C_{\infty}\left(\delta_{o p t}, \xi, \varphi\right)$

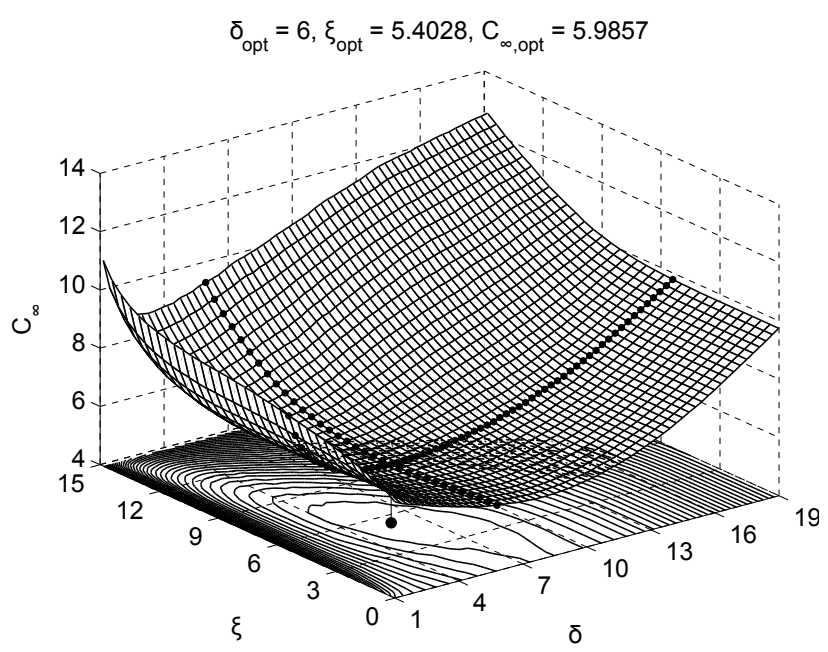

(d) $C_{\infty}\left(\delta, \xi, \varphi_{\text {opt }}\right)$

Figure 9: Illustration of the stationary law and the cost rate of the system maintained under the $(\delta, \xi, \varphi)$ policy

where $C_{\infty}$ and $C_{\infty, \text { opt }}$ are the long-run expected maintenance cost rate of the policy in its non-optimal and optimal configurations respectively. Obviously, the higher the value of $\epsilon_{C}(\%)$, the less the policy is robust.

All numerical experiments represented in this section are based on the Gamma deterioration process-based failure model introduced in Section II-A

\section{A. Performance Analysis}

We study at first the impact of the maintenance costs on the performance of the proposed predictive maintenance decision framework. To this end, we set the system characteristics at $\alpha=0.2, \beta=0.2$ (i.e., $m=1, \sigma^{2}=5$ ) and $L=15$, and the practical constraint $C_{i}<C_{p}<C_{c}$ leads us to take $C_{c}=100$ and consider the three cases

1) varied inspection cost: $C_{p}=50, C_{d}=25$, and $C_{i}$ varies from 1 to 49 with step 1 ,

2) varied PR cost: $C_{i}=5, C_{d}=25$, and $C_{p}$ varies from 6 to 99 with step 3 ,

3) varied downtime cost rate: $C_{i}=5, C_{p}=50$, and $C_{d}$ varies from 10 to 190 with step 5 .

Next, we study the impact of the deterioration variance $\sigma^{2}$. To this end, we take $L=15, m=1$ and vary $\sigma^{2}$ from 1 to 19 with step 1; the set of maintenance costs is fixed at $C_{i}=5, C_{p}=50, C_{c}=100$ and $C_{d}=25$. For each of the above cases, we sketch the relative gains $\kappa_{C}(\%)$ of the policies $(\delta, \xi, \lambda),(\delta, \xi, \varphi)$ and $(\delta, \xi, \eta)$ compared with the $(\delta, \zeta)$ policy, and the obtained results are shown in Figure 11. The upper curves in the figure correspond to the maintenance policies with higher performance.

Unsurprisingly, the proposed predictive maintenance decision framework is always more profitable than the classical one since $\kappa_{C}(\%) \geq 0$. So, there is no risk in using this framework (i.e., it returns to the traditional framework in the worst case). In reality, the performance of the new framework increases with higher inspection costs, and this is especially significant when the inspection is expensive (see Figure 11a) as it can avoid inopportune inspections. When setting the inspection cost $C_{i}$ and increasing the PR 

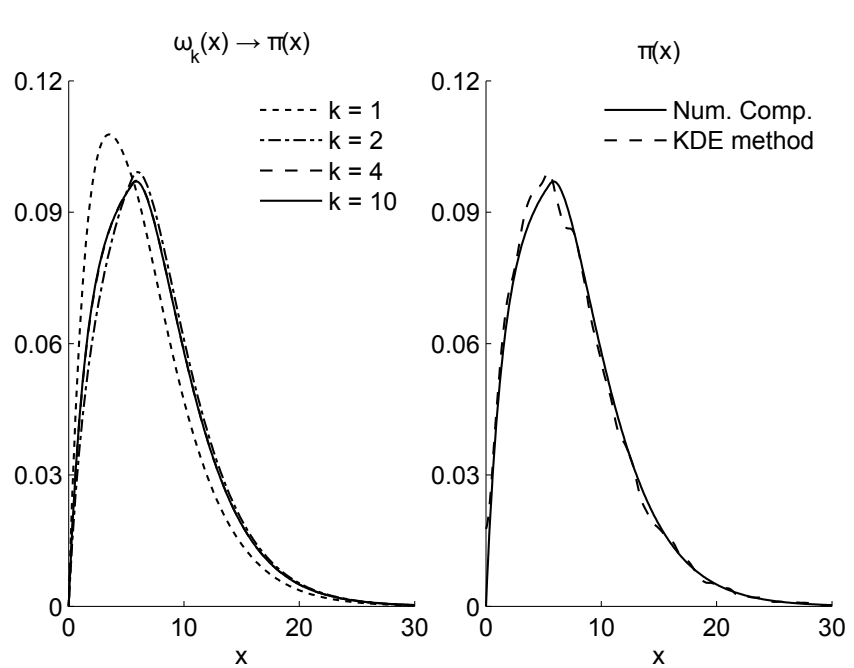

(a) $\omega_{k}(x)$ and $\pi(x)$

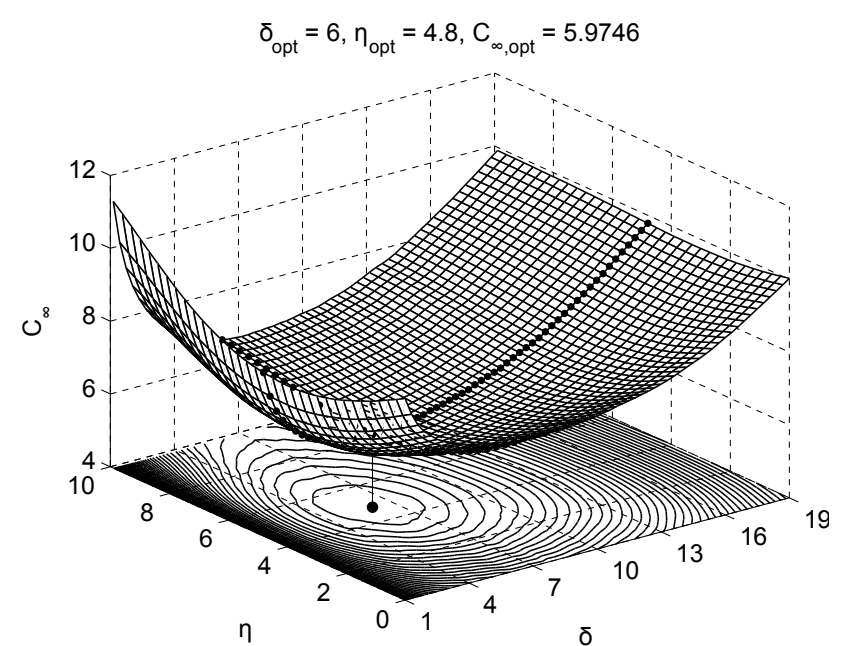

(c) $C_{\infty}\left(\delta, \xi_{\text {opt }}, \eta\right)$

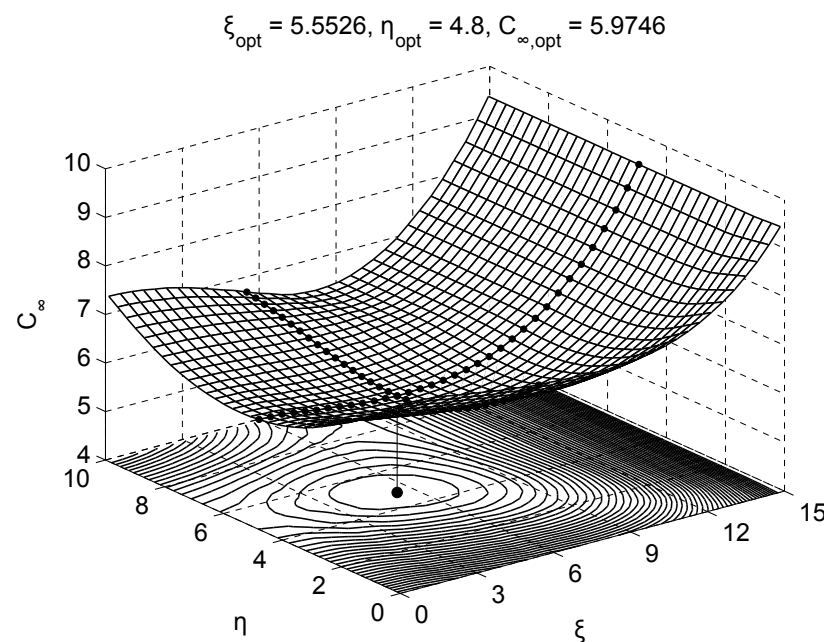

(b) $C_{\infty}\left(\delta_{o p t}, \xi, \eta\right)$

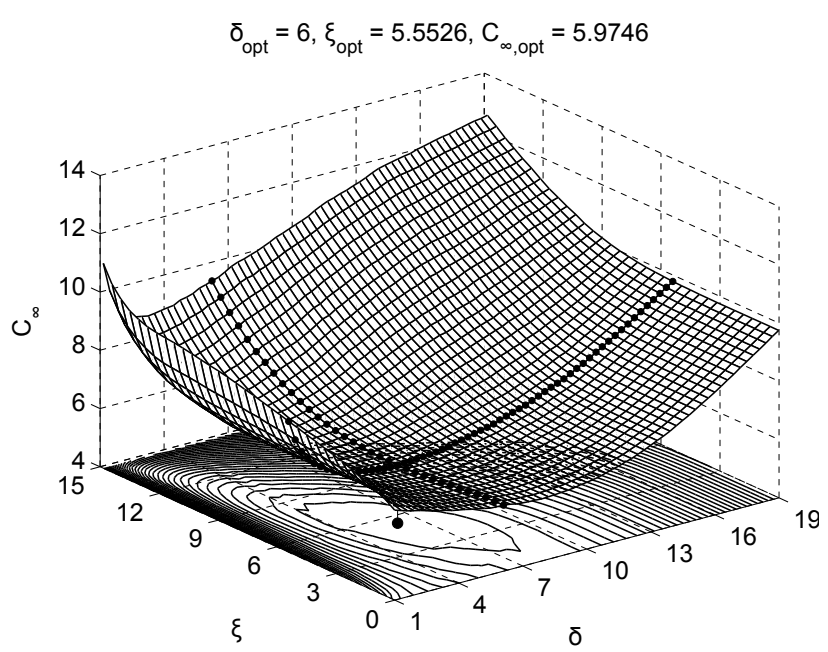

(d) $C_{\infty}\left(\delta, \xi, \eta_{o p t}\right)$

Figure 10: Illustration of the stationary law and the cost rate of the system maintained under the $(\delta, \xi, \eta)$ policy

cost $C_{p}$, the relative gains $\kappa_{C}(\%)$ associated with the $(\delta, \xi, \varphi)$ policy and the $(\delta, \xi, \eta)$ policy are decreasing (see Figure $11 \mathrm{~b}$ ). This is because $C_{i}$ becomes relatively smaller than $C_{p}$, which leads to a weaker $\kappa_{C}(\%)$. For the third case, the relative gains are more or less constant with respect to $C_{d}$ (see Figure 11c). These phenomena show that, unlike the inspection cost, the PR cost and the downtime cost rate do not affect much the performance of the proposed framework.

Looking at Figure 11d, the relative gain $\kappa_{C}(\%)$ of the $(\delta, \xi, \lambda)$ policy compared with the $(\delta, \zeta)$ policy is almost constant with respect to the deterioration variance $\sigma^{2}$. This is because the waiting time $\psi$, which causes main difference between these two policies, is fixed regardless of the system deterioration state, and therefore is not affected by its variance. For the $(\delta, \xi, \varphi)$ policy and the $(\delta, \xi, \eta)$ policy, the waiting times $\psi$ depend closely on the system deterioration state, and the sooner the precision level of RUL prognosis is reached, the less inopportune inspections are performed. The lower deterioration variance allows a RUL prognosis with higher precision, hence these two policies are most profitable at the small values of $\sigma^{2}$, and their profit decreases when $\sigma^{2}$ increases. For large values of $\sigma^{2}$, the performances of the three considered policies $(\delta, \xi, \lambda),(\delta, \xi, \varphi)$ and $(\delta, \xi, \eta)$ come close (see Figure 11d). This phenomenon can be explained by the fact that the system deterioration becomes very chaotic in this case, the adaptive waiting times $\psi$ of the $(\delta, \xi, \varphi)$ policy and the $(\delta, \xi, \eta)$ policy cannot be in tune with the variation of the system condition. As such, instead of relying on the deterioration states detected at inspection times, these waiting times have to be based on their average value. In other words, the $(\delta, \xi, \varphi)$ policy and the $(\delta, \xi, \eta)$ policy return to the $(\delta, \xi, \lambda)$ policy, and their performances become almost identical.

Figure 11 also shows the efficiency of the different approaches to build the waiting time $\psi$. Obviously, the condition-based approach can lead to more savings in the maintenance costs than the time-based approach in most situations, as it is adaptive and follows closely the system state. The two proposed ways of determining the condition-based PR waiting time $\psi$ (i.e., reliability-based waiting time (also known as RUL-based waiting time) and MRL-based waiting time) give similar profits under their optimal configuration. This is not very surprising because the criterion of interest is the long-run expected maintenance cost rate (13), and from this average 


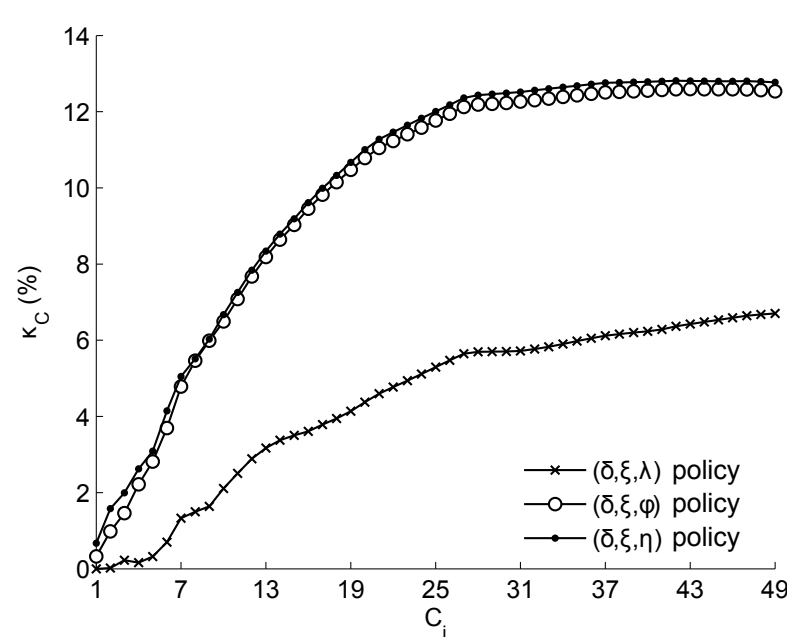

(a) $C_{i}$ varies

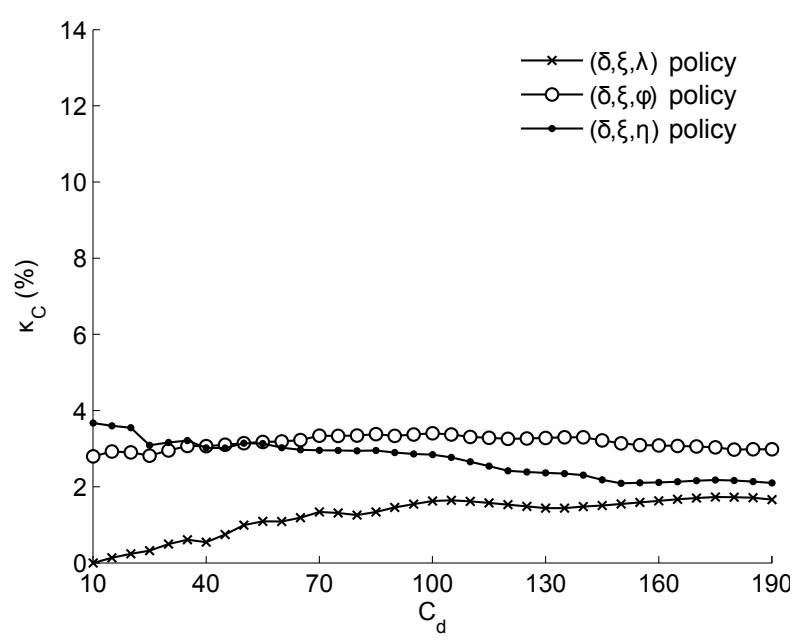

(c) $C_{d}$ varies

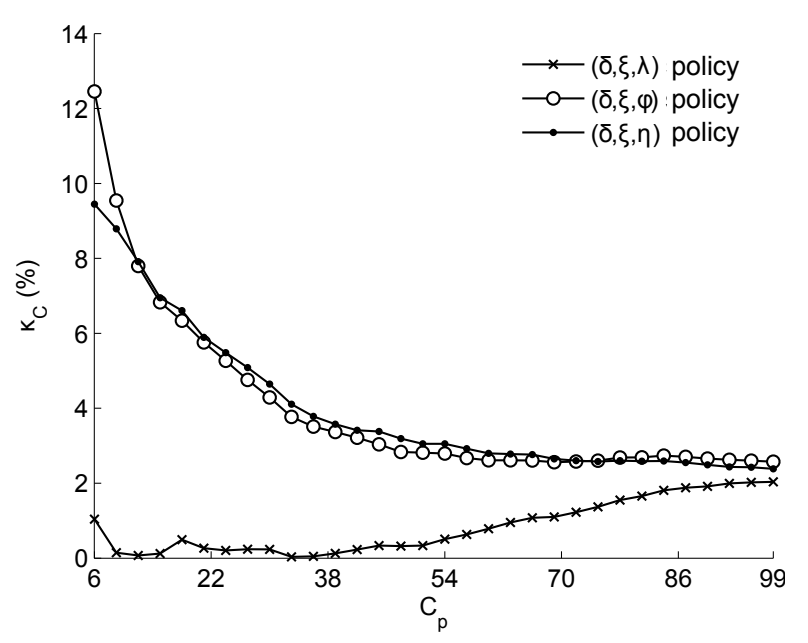

(b) $C_{p}$ varies

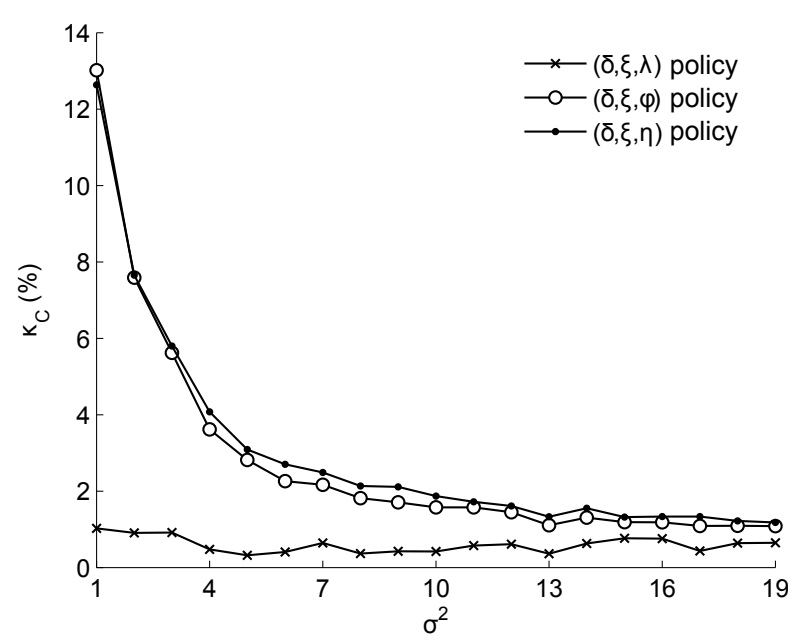

(d) $\sigma^{2}$ varies

Figure 11: $\kappa_{C}(\%)$ under various configurations of maintenance costs and of deterioration variances

viewpoint, the system MRL contains all the information about the system RUL. In more details, the reliability-based waiting time seems slightly better when the downtime cost rate $C_{d}$ is higher (see Figure 11c), because it controls more efficiently the system availability.

\section{B. Robustness Analysis}

Besides the performance, the robustness is another essential criterion to assess the effectiveness of a maintenance decision framework. In reality, making a maintenance decision is always confronted with uncertainties owing to the lack of data, measurement errors, variability, modeling uncertainties, etc. This leads to errors in determining the optimal decisions of a maintenance policy, and hence a loss in its performance. A robust policy should have the ability to resist such errors. This section focuses on determining which one among the 3 proposed policies $(\delta, \xi, \lambda),(\delta, \xi, \varphi)$ and $(\delta, \xi, \eta)$ is robust. Since the only difference between these policies is the way to determine the waiting time, it is sufficient to assess their robustness by analyzing the impact of the suboptimal choice of the waiting time thresholds on the maintenance cost rate. By this way, we fix the inspection period $\delta$ and the deterioration threshold associated with the RUL prognosis precision $\xi$ at their optimal values, vary the other decision parameters (i.e., $\lambda$ for the $(\delta, \xi, \lambda)$ policy, $\varphi$ for the $(\delta, \xi, \varphi)$ policy, and $\eta$ for the $(\delta, \xi, \eta)$ policy) around their optimal values with a relative error $\epsilon_{d}(\%)$ varied from $-20 \%$ to $+20 \%$, and we investigate the evolution of $\epsilon_{C}(\%)$ under various configurations of system characteristics. Specifically, the study is based on the set of parameters $L=15, C_{i}=5, C_{p}=70, C_{c}=100$ and $C_{d}=25$, and the 2 following configurations of system behaviors are considered

1) small variance rate: $\sigma^{2}=3, m=1$ (i.e., $\alpha=1 / 3, \beta=1 / 3$ ),

2) high variance rate: $\sigma^{2}=9, m=1$ (i.e., $\alpha=1 / 9, \beta=1 / 9$ ).

The results are shown in Figure 12. Not unexpectedly, the $(\delta, \xi, \lambda)$ policy is the most robust and the $(\delta, \xi, \varphi)$ policy is the least robust, because their waiting times are respectively unadapted and closely adapted to the system deterioration. Compared to the $(\delta, \xi, \varphi)$ 


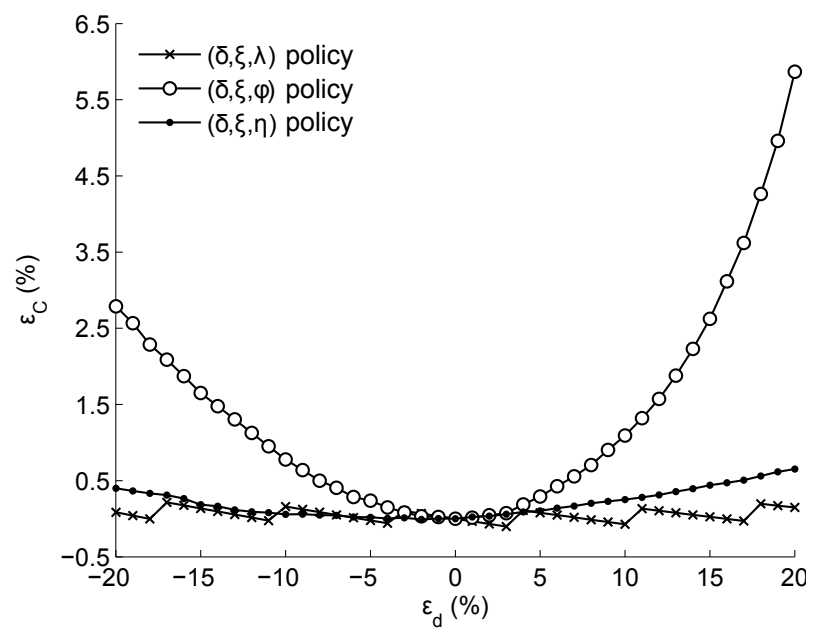

(a) $\sigma^{2}=3$

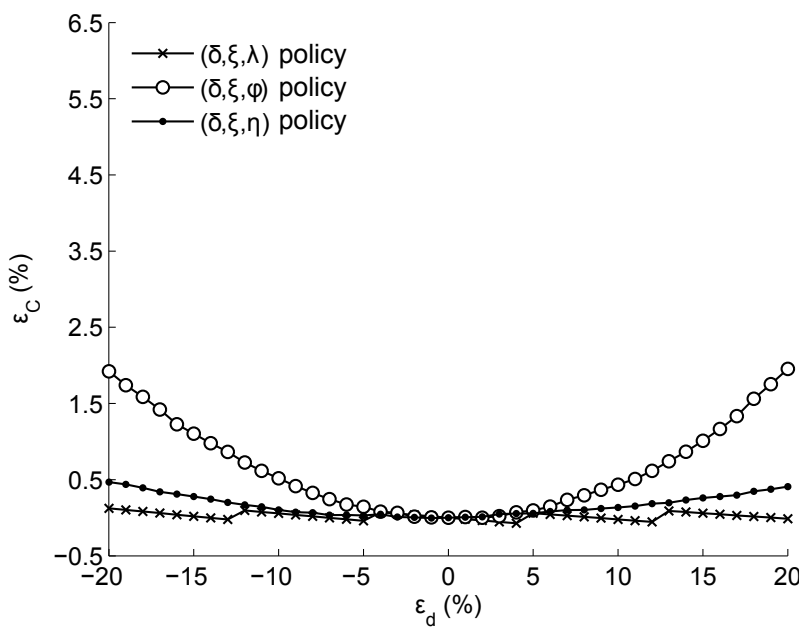

(b) $\sigma^{2}=9$

Figure 12: $\epsilon_{C}(\%)$ under various configurations of deterioration variances

policy, the $(\delta, \xi, \eta)$ policy has a remarkable robustness level, because the nature of the decision variable $\eta$ is not sensitive to the change of the system deterioration (see also the end of Section III-D). We can understand more easily this point by observing Figure 13. This figure represents the evolution of expected waiting times of the 3 considered policies with respect to $\epsilon_{d}(\%)$. Looking at the

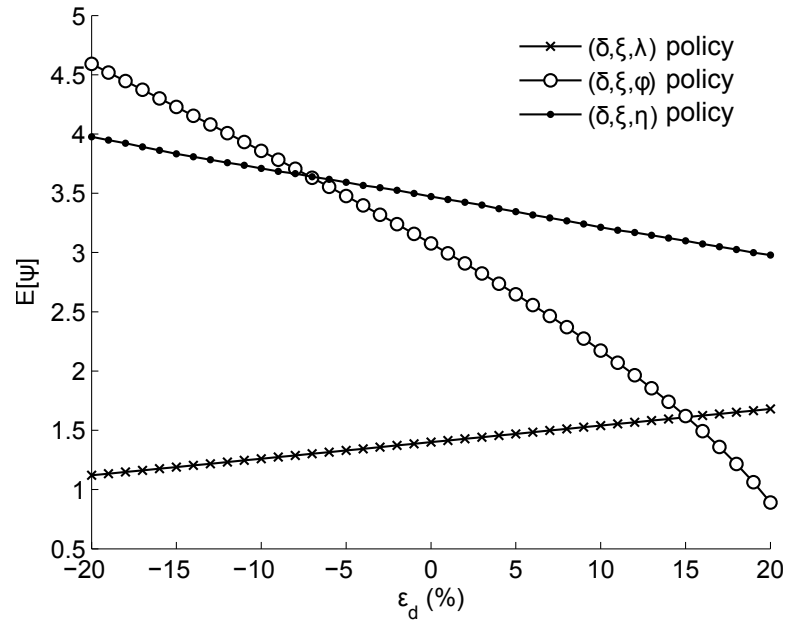

(a) $\sigma^{2}=3$

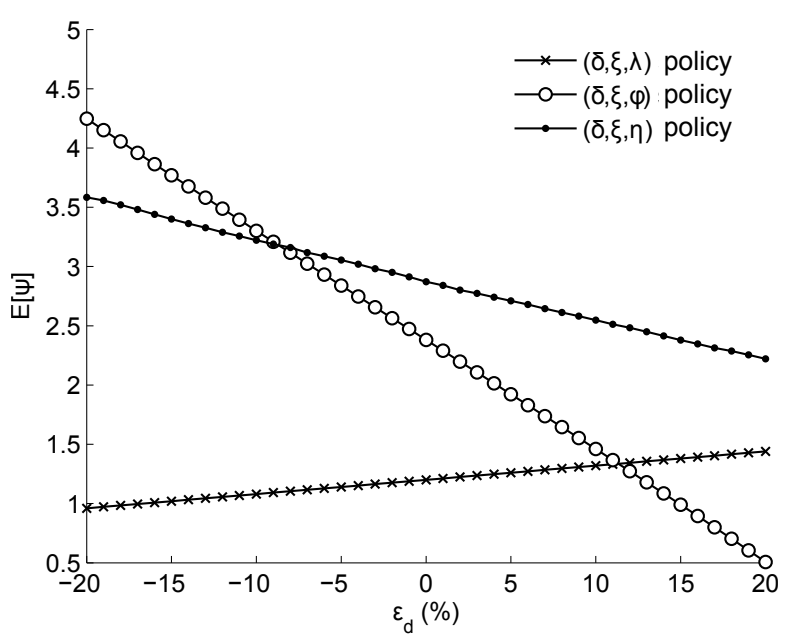

(b) $\sigma^{2}=9$

Figure 13: $E[\psi]$ under various configurations of deterioration variances

slopes of these curves, the variability of the expected waiting time in the $(\delta, \xi, \eta)$ policy is much smaller than in the $(\delta, \xi, \varphi)$ policy, so the $(\delta, \xi, \eta)$ policy is more robust. Moreover, from Figure 13a and Figure 13b, we also see that the variance of the deterioration process does not influence much the robustness of the $(\delta, \xi, \lambda)$ and the $(\delta, \xi, \eta)$ policies; while the robustness of the $(\delta, \xi, \varphi)$ policy is improved for higher deterioration variance thanks to the fact that the system conditional reliability becomes less sensitive to the deterioration in this case. This is even more impressive knowing that the system conditional MRL resists the variability of deterioration much worse than the system conditional reliability (see Figure 3). As a conclusion, the waiting time setting used in the $(\delta, \xi, \eta)$ policy can assure not only the performance but also the robustness of a maintenance policy.

\section{Vi. Conclusion \& Perspectives}

In this work, we propose and assess a new parametric predictive maintenance decision framework considering improved health prognosis precision for stochastically deteriorating single-unit systems. Within this new framework, statistical measures representing the variability in the system RUL estimation and the location of the RUL distribution are used to decide and schedule separately inspections and replacements respectively. This allows to break the inherent drawback of the classical "state-of-the-art" predictive maintenance framework. Several numerical experiments show the advantage of the proposed maintenance decision framework compared to the traditional one. In fact, there is no risk in using this new framework, because it is more general, more flexible, and 
returns to the traditional framework in the worse case. Furthermore, the new framework leads to significantly improved maintenance performance for systems with small deterioration variance and high inspection costs. The results from the numerical experiments also confirm the interest of the prognostics information on the system health for maintenance decision-making and, at the same time, emphasize the necessity of using this information in a proper way. These conclusions support the momentum towards investing in prognostics and health management technologies, and building new predictive maintenance policies.

The work presented in this paper is mainly theoretical, and even if the theoretical results are encouraging, the next step to study the applicability of the proposed approach is of course to gather further evidences of their practical interests from field experiments. Consequently, one of our perspectives is to validate the proposed maintenance framework with real data, which requires the identification of a suitable system, the implementation of condition monitoring systems able to deliver the required data and further data analysis for deterioration modeling. For example, a phase of parameters estimation for the deterioration-based failure model should be implemented before going further with the maintenance decision-making approach. Regarding the implementation of the proposed maintenance decision rule, it is noteworthy that despite long mathematical developments and numerical procedures to derive the optimal tuning of the proposed policies, in the end, these policies remains simple to implement in practice. Indeed, they use periodic inspection schemes and control-limit decision rules (i.e. simple comparison to a threshold). We have chosen on purpose to work with this kind of structures for the maintenance decision rule because, even if they might not be absolutely optimal, they are easy to implement. Other perspectives will be devoted to the proposition, characterization and evaluation of predictive maintenance decision rules for multi-unit systems (e.g., $k$-out-of- $n$ deteriorating systems). From the application point of view, we think that the proposed framework can be used to build efficient maintenance policies adapted to remote systems with limited maintenance resources and facilities (e.g., offshore systems such as submarine power cables, offshore wind turbines, subsea blowout preventer system, etc.).

\section{APPENDIX}

\section{Proof of (9)}

The standard deviation $\vartheta\left(\tau_{i} \mid X_{\tau_{i}}\right)$ at time $\tau_{i}$ given the deterioration level $X_{\tau_{i}}=x$ can be computed as

$$
\vartheta\left(\tau_{i} \mid X_{\tau_{i}}=x\right)=\sqrt{E\left[\left(\rho\left(\tau_{i} \mid X_{\tau_{i}}=x\right)-\mu\left(\tau_{i} \mid X_{\tau_{i}}=x\right)\right)^{2}\right]}=\sqrt{E\left[\rho^{2}\left(\tau_{i} \mid x\right)\right]-\mu^{2}\left(\tau_{i} \mid x\right)},
$$

where $\rho\left(\tau_{i} \mid x\right)$ and $\mu\left(\tau_{i} \mid x\right)$ denote the associated system RUL and system MRL respectively. Since for any differentiable function $h(u)$ such that $E[h(U)]$ exists, where $U$ is a random variable and $U \geq 0$, then

$$
E[h(U)]=-\int_{0}^{\infty} h(u) d R(u)=-[h(u) R(u)]_{0}^{\infty}+\int_{0}^{\infty} R(u) d h(u)=h(0)+\int_{0}^{\infty} R(u) d h(u),
$$

where $R(u)$ denotes the survival function of $U$. Applying (30) for $U=\rho\left(\tau_{i} \mid X_{\tau_{i}}=x\right)$ and $h(u)=u^{2}$, then

$$
E\left[\rho^{2}\left(\tau_{i} \mid X_{\tau_{i}}=x\right)\right]=2 \int_{0}^{\infty} u F_{\alpha u, \beta}(L-x) d u .
$$

As a result, the standard deviation $\vartheta\left(\tau_{i} \mid X_{\tau_{i}}\right)$ is obtained by

$$
\vartheta\left(\tau_{i} \mid X_{\tau_{i}}=x\right)=\sqrt{2 \int_{0}^{\infty} u F_{\alpha u, \beta}(L-x) d u-\mu^{2}\left(\tau_{i} \mid X_{\tau_{i}}=x\right)} .
$$

\section{ACKNOWLEDGMENT}

This work has been partially supported by the LabEx PERSYVAL-Lab (ANR-11-LABX-0025-01) funded by the French program Investissement d'avenir

\section{REFERENCES}

[1] J. McCall, "Maintenance policies for stochastically failing equipment: a survey," Management science, vol. 11, no. 5, pp. 493$524,1965$.

[2] H. Wang, "A survey of maintenance policies of deteriorating systems," European Journal of Operational Research, vol. 139, no. 3, pp. 469-489, 2002.

[3] J. Shin and H. Jun, "On condition based maintenance policy," Journal of Computational Design and Engineering, vol. 2, no. 2, pp. 119-127, 2015. 
[4] H. Iqbal, S. Tesfamariam, H. Haider, and R. Sadiq, "Inspection and maintenance of oil \& gas pipelines: a review of policies," Structure and Infrastructure Engineering, vol. 13, no. 6, pp. 794-815, 2017.

[5] A. Jardine, D. Lin, and D. Banjevic, "A review on machinery diagnostics and prognostics implementing condition-based maintenance," Mechanical systems and signal processing, vol. 20, no. 7, pp. 1483-1510, 2006.

[6] A. Bousdekis, B. Magoutas, D. Apostolou, and G. Mentzas, "Review, analysis and synthesis of prognostic-based decision support methods for condition based maintenance," Journal of Intelligent Manufacturing, pp. 1-14, 2016.

[7] R. Ahmad and S. Kamaruddin, "A review of condition-based maintenance decision-making," European Journal of Industrial Engineering, vol. 6, no. 5, pp. 519-541, 2012.

[8] J. Van Noortwijk, "A survey of the application of gamma processes in maintenance," Reliability Engineering \& System Safety, vol. 94, no. 1, pp. 2-21, 2009.

[9] W. Wang, "Overview of a semi-stochastic filtering approach for residual life estimation with applications in condition based maintenance," Proceedings of the Institution of Mechanical Engineers, Part O: Journal of Risk and Reliability, vol. 225, no. 2, pp. 185-197, 2011.

[10] W. Wang, “An overview of the recent advances in delay-time-based maintenance modelling," Reliability Engineering \& System Safety, vol. 106, pp. 165-178, 2012.

[11] R. Ahmad and S. Kamaruddin, "An overview of time-based and condition-based maintenance in industrial application," Computers \& Industrial Engineering, vol. 63, no. 1, pp. 135-149, 2012.

[12] M. Sánchez-Silva, D. Frangopol, J. Padgett, and M. Soliman, "Maintenance and operation of infrastructure systems: Review," Journal of Structural Engineering, vol. 142, no. 9, pp. F4016004-1-16, 2016.

[13] L. Wang, J. Chu, and W. Mao, "A condition-based replacement and spare provisioning policy for deteriorating systems with uncertain deterioration to failure," European Journal of Operational Research, vol. 194, no. 1, pp. 184-205, 2009.

[14] D. Frangopol, M. Kallen, and J. Van Noortwijk, "Probabilistic models for life-cycle performance of deteriorating structures: review and future directions," Progress in Structural Engineering and Materials, vol. 6, no. 4, pp. 197-212, 2004.

[15] C. Bérenguer, C. Chu, and A. Grall, "Inspection and maintenance planning: An application of semi-markov decision processes," Journal of Intelligent Manufacturing, vol. 8, no. 5, pp. 467-476, 1997.

[16] C. Chu, J. Proth, and P. Wolff, "Predictive maintenance: the one-unit replacement model," International Journal of Production Economics, vol. 54, no. 3, pp. 285-295, 1998.

[17] M. Newby and C. Barker, "A bivariate process model for maintenance and inspection planning," International Journal of Pressure Vessels and Piping, vol. 83, no. 4, pp. 270-275, 2006.

[18] K. Papakonstantinou and M. Shinozuka, "Planning structural inspection and maintenance policies via dynamic programming and markov processes. part i: Theory," Reliability Engineering \& System Safety, vol. 130, pp. 202-213, 2014.

[19] K. Papakonstantinou and M. Shinozuka, "Planning structural inspection and maintenance policies via dynamic programming and markov processes. part ii: Pomdp implementation,” Reliability Engineering \& System Safety, vol. 130, pp. $214-224,2014$.

[20] A. Grall, L. Dieulle, C. Bérenguer, and M. Roussignol, "Continuous-time predictive-maintenance scheduling for a deteriorating system," IEEE Transactions on Reliability, vol. 51, no. 2, pp. 141-150, 2002.

[21] B. Castanier, A. Grall, and C. Bérenguer, "A condition-based maintenance policy with non-periodic inspections for a two-unit series system," Reliability Engineering \& System Safety, vol. 87, no. 1, pp. 109-120, 2005.

[22] L. Wang, J. Chu, and W. Mao, "A condition-based order-replacement policy for a single-unit system," Applied mathematical modelling, vol. 32, no. 11, pp. 2274-2289, 2008.

[23] E. Deloux, B. Castanier, and C. Bérenguer, "Predictive maintenance policy for a gradually deteriorating system subject to stress," Reliability Engineering \& System Safety, vol. 94, no. 2, pp. 418-431, 2009.

[24] K. Huynh, A. Barros, and C. Bérenguer, "Multi-level decision-making for the predictive maintenance of k-out-of-n:f deteriorating systems," IEEE Transactions on Reliability, vol. 64, no. 1, pp. 94-117, 2015.

[25] C. Bérenguer, "On the mathematical condition-based maintenance modelling for continuously deteriorating systems," International Journal of Materials and Structural Reliability, vol. 6, no. 2, pp. 133-151, 2008.

[26] C. Bérenguer, A. Grall, L. Dieulle, and M. Roussignol, "Maintenance policy for a continuously monitored deteriorating system," Probability in the Engineering and Informational Sciences, vol. 17, no. 02, pp. 235-250, 2003.

[27] H. Liao, E. Elsayed, and L. Chan, "Maintenance of continuously monitored degrading systems," European Journal of Operational Research, vol. 175, no. 2, pp. 821-835, 2006.

[28] A. Grall, L. Dieulle, C. Bérenguer, and M. Roussignol, "Asymptotic failure rate of a continuously monitored system,” Reliability 
Engineering \& System Safety, vol. 91, no. 2, pp. 126-130, 2006.

[29] K. Huynh, A. Barros, C. Bérenguer, and I. Castro, "A periodic inspection and replacement policy for systems subject to competing failure modes due to degradation and traumatic events," Reliability Engineering \& System Safety, vol. 96, no. 04, pp. 497-508, 2011.

[30] E. Deloux, M. Fouladirad, and C. Bérenguer, "Health-and-usage-based maintenance policies for a partially observable deteriorating system," Proceedings of the Institution of Mechanical Engineers, Part O: Journal of Risk and Reliability, vol. 230, no. 1, pp. 120-129, 2016.

[31] L. Cui, M. Xie, and H. Loh, "Inspection schemes for general systems," IIE Transactions, vol. 36, no. 9, pp. 817-825, 2004.

[32] H. Golmakani, "Optimal age-based inspection scheme for condition-based maintenance using a* search algorithm," International Journal of Production Research, vol. 50, no. 23, pp. 7068-7080, 2012.

[33] A. Ponchet, M. Fouladirad, and A. Grall, "Assessment of a maintenance model for a multi-deteriorating mode system," Reliability Engineering \& System Safety, vol. 95, no. 11, pp. 1244-1254, 2010.

[34] A. Grall, C. Bérenguer, and L. Dieulle, "A condition-based maintenance policy for stochastically deteriorating systems," Reliability Engineering \& System Safety, vol. 76, no. 2, pp. 167-180, 2002.

[35] Y. Yang and G. Klutke, "Improved inspection schemes for deteriorating equipment," Probability in the Engineering and Informational Sciences, vol. 14, no. 4, pp. 445-460, 2000.

[36] K. Huynh, A. Barros, and C. Bérenguer, "Maintenance decision-making for systems operating under indirect condition monitoring: Value of online information and impact of measurement uncertainty," IEEE Transactions on Reliability, vol. 61, no. 2, pp. 410-425, 2012.

[37] K. Huynh, A. Barros, and C. Bérenguer, "Adaptive condition-based maintenance decision framework for deteriorating systems operating under variable environment and uncertain condition monitoring," Proceedings of the Institution of Mechanical Engineers, Part O: Journal of Risk and Reliability, vol. 226, no. 6, pp. 602-623, 2012.

[38] T. Nakagawa, S. Mizutani, and M. Chen, "A summary of periodic and random inspection policies," Reliability Engineering \& System Safety, vol. 95, no. 8, pp. 906-911, 2010.

[39] K. Huynh, I. Castro, A. Barros, and C. Bérenguer, "Modeling age-based maintenance strategies with minimal repairs for systems subject to competing failure modes due to degradation and shocks," European Journal of Operational Research, vol. 218, no. 1, pp. 140-151, 2012.

[40] K. Huynh, I. Castro, A. Barros, and C. Bérenguer, "On the use of mean residual life as a condition index for condition-based maintenance decision-making," IEEE Transactions on Systems, Man, and Cybernetics: Systems, vol. 44, no. 7, pp. 877-893, 2014.

[41] M. Zhang, O. Gaudoin, and M. Xie, "Degradation-based maintenance decision using stochastic filtering for systems under imperfect maintenance," European Journal of Operational Research, vol. 245, no. 2, pp. 531-541, 2015.

[42] T. Aven, "Condition based replacement policies - a counting process approach," Reliability Engineering \& System Safety, vol. 51, no. 3, pp. 275-281, 1996.

[43] M. Ouali, L. Tadj, S. Yacout, and D. Ait-Kadi, "A survey of replacement models with minimal repair," in Replacement Models with Minimal Repair (L. Tadj, M.-S. Ouali, S. Yacout, and D. Ait-Kadi, eds.), pp. 3-100, Springer, 2011.

[44] H. Pham and H. Wang, "Imperfect maintenance," European journal of operational research, vol. 94, no. 3, pp. 425-438, 1996.

[45] B. Castanier, C. Bérenguer, and A. Grall, "A sequential condition-based repair/replacement policy with non-periodic inspections for a system subject to continuous wear," Applied stochastic models in business and industry, vol. 19, no. 4, pp. 327-347, 2003.

[46] B. Saassouh, L. Dieulle, and A. Grall, "Online maintenance policy for a deteriorating system with random change of mode," Reliability Engineering \& System Safety, vol. 92, no. 12, pp. 1677-1685, 2007.

[47] H. Kawai, J. Koyanagi, and M. Ohnishi, "Optimal maintenance problems for markovian deteriorating systems," in Stochastic Models in Reliability and Maintenance, pp. 193-218, Springer, 2002.

[48] M. Abdel-Hameed, Lévy Processes and Their Applications in Reliability and Storage. SpringerBriefs in Statistics, Springer, 2014.

[49] B. Lee, C. Kang, S. Kim, and S. Bae, "Optimal replacement strategy for stochastic deteriorating system with random wear limit under periodic inspections," The International Journal of Advanced Manufacturing Technology, vol. 71, no. 1-4, pp. 219-231, 2014.

[50] M. S. Abdel-Hameed, "Optimal predictive maintenance policies for a deteriorating system: the total discounted cost and the long-run average cost cases," Communications in Statistics - Theory and Methods, vol. 33, no. 3, pp. 735-745, 2004. 
[51] J. van der Weide, M. Pandey, and J. van Noortwijk, "Discounted cost model for condition-based maintenance optimization," Reliability Engineering \& System Safety, vol. 95, no. 3, pp. 236-246, 2010.

[52] Y. Wang and H. Pham, "Maintenance modeling and policies," in Stochastic Reliability and Maintenance Modeling, pp. 141-158, Springer, 2013.

[53] E. Khoury, E. Deloux, A. Grall, and C. Bérenguer, "On the use of time-limited information for maintenance decision support: A predictive approach under maintenance constraints," Mathematical Problems in Engineering, vol. 2013, 2013. Article ID 983595, 11 pages, doi:10.1155/2013/983595.

[54] K. Le Son, M. Fouladirad, and A. Barros, "Remaining useful lifetime estimation and noisy gamma deterioration process," Reliability Engineering \& System Safety, vol. 149, pp. 76-87, 2016.

[55] K. Nguyen, P. Do, and A. Grall, "Condition-based maintenance for multi-component systems using importance measure and predictive information," International Journal of Systems Science: Operations \& Logistics, vol. 1, no. 4, pp. 228-245, 2014.

[56] W. Zhu, M. Fouladirad, and C. Bérenguer, "Condition-based maintenance policies for a combined wear and shock deterioration model with covariates," Computers \& Industrial Engineering, vol. 85, pp. 268-283, 2015.

[57] H. Li, E. Deloux, and L. Dieulle, "A condition-based maintenance policy for multi-component systems with lévy copulas dependence," Reliability Engineering \& System Safety, vol. 149, pp. 44-55, 2016.

[58] K. Huynh, A. Grall, and C. Bérenguer, "Assessment of diagnostic and prognostic condition indices for efficient and robust maintenance decision-making of systems subject to stress corrosion cracking," Reliability Engineering \& System Safety, vol. 159, pp. 237-254, 2017.

[59] C. Cocozza-Thivent, Processus stochastiques et fiabilité des systèmes, vol. 28 of Mathématiques \& Applications. Springer, 1997. In French.

[60] Z. Xu, Y. Ji, and D. Zhou, "Real-time reliability prediction for a dynamic system based on the hidden degradation process identification," IEEE Transactions on Reliability, vol. 57, no. 2, pp. 230-242, 2008.

[61] Z. Xu, Y. Ji, and D. Zhou, "A new real-time reliability prediction method for dynamic systems based on on-line fault prediction," IEEE Transactions on Reliability, vol. 58, no. 3, pp. 523-538, 2009.

[62] D. Nguyen, L. Dieulle, and A. Grall, "Remaining useful lifetime prognosis of controlled systems: A case of stochastically deteriorating actuator," Mathematical Problems in Engineering, 2015. Article ID 356916, in press.

[63] N. Singpurwalla, "Survival in dynamic environments," Statistical Science, vol. 10, no. 1, pp. 86-103, 1995.

[64] X. Si, W. Wang, C. Hu, and D. Zhou, "Remaining useful life estimation - a review on the statistical data driven approaches," European Journal of Operational Research, vol. 213, no. 1, pp. 1-14, 2011.

[65] N. Bousquet, M. Fouladirad, A. Grall, and C. Paroissin, "Bayesian gamma processes for optimizing condition-based maintenance under uncertainty," Applied Stochastic Models in Business and Industry, vol. 31, no. 3, pp. 360-379, 2015.

[66] X. Wang, "Nonparametric estimation of the shape function in a gamma process for degradation data," Canadian Journal of Statistics, vol. 37, no. 1, pp. 102-118, 2009.

[67] M. Kallen and J. van Noortwijk, “Optimal maintenance decisions under imperfect inspection,” Reliability Engineering \& System Safety, vol. 90, no. 2-3, pp. 177-185, 2005.

[68] T. Santini, S. Morand, M. Fouladirad, L. Phung, F. Miller, B. Foucher, A. Grall, and B. Allard, "Accelerated degradation data of sic mosfets for lifetime and remaining useful life assessment," Microelectronics Reliability, vol. 54, no. 9, pp. 1718-1723, 2014.

[69] Y. Langeron, A. Grall, and A. Barros, "A modeling framework for deteriorating control system and predictive maintenance of actuators," Reliability Engineering \& System Safety, vol. 140, pp. 22-36, 2015.

[70] C. Blain, A. Barros, A. Grall, and Y. Lefebvre, "Modelling of stress corrosion cracking with stochastic processes-application to steam generators," in Pro. of the European Safety and Reliability Conference - ESREL 2007, pp. 2395-2400, 2007.

[71] A. Lehmann, "Joint modeling of degradation and failure time data," Journal of Statistical Planning and Inference, vol. 139, no. 5, pp. 1693-1706, 2009.

[72] Y. Peng, M. Dong, and M. Zuo, "Current status of machine prognostics in condition-based maintenance: a review," The International Journal of Advanced Manufacturing Technology, vol. 50, no. 1-4, pp. 297-313, 2010.

[73] J. Zaytoon and S. Lafortune, "Overview of fault diagnosis methods for discrete event systems," Annual Reviews in Control, vol. 37, no. 2, pp. 308-320, 2013.

[74] L. Travé-Massuyès, "Bridging control and artificial intelligence theories for diagnosis: A survey," Engineering Applications of Artificial Intelligence, vol. 27, pp. 1-16, 2014. 
[75] I. Lopez and N. Sarigul-Klijn, "A review of uncertainty in flight vehicle structural damage monitoring, diagnosis and control: Challenges and opportunities," Progress in Aerospace Sciences, vol. 46, no. 7, pp. 247-273, 2010.

[76] R. Ahmadi, "A new approach to modeling condition-based maintenance for stochastically deteriorating systems," International Journal of Reliability, Quality and Safety Engineering, vol. 21, no. 05, p. 1450024, 2014.

[77] J. Sikorskaa, M. Hodkiewicz, and L. Ma, "Prognostic modelling options for remaining useful life estimation by industry," Mechanical Systems and Signal Processing, vol. 25, no. 5, pp. 1803-1836, 2011.

[78] L. Liao and F. Kottig, "Review of hybrid prognostics approaches for remaining useful life prediction of engineered systems, and an application to battery life prediction," IEEE Transactions on Reliability, vol. 63, no. 1, pp. 191-207, 2014.

[79] M. Kan, A. Tan, and J. Mathew, "A review on prognostic techniques for non-stationary and non-linear rotating systems," Mechanical Systems and Signal Processing, vol. 62, pp. 1-20, 2015.

[80] Z. Zhang, X. Si, C. Hu, and X. Kong, "Degradation modeling-based remaining useful life estimation: A review on approaches for systems with heterogeneity," Proceedings of the Institution of Mechanical Engineers, Part O: Journal of Risk and Reliability, vol. 229, no. 4, pp. 343-355, 2015.

[81] K. Le Son, M. Fouladirad, A. Barros, E. Levrat, and B. Iung, "Remaining useful life estimation based on stochastic deterioration models: A comparative study," Reliability Engineering \& System Safety, vol. 112, pp. 165-175, 2013.

[82] K. Park, “Optimal continuous-wear limit replacement under periodic inspections," IEEE Transactions on Reliability, vol. 37, no. 1, pp. 97-102, 1988.

[83] X. Zhao, M. Fouladirad, C. Bérenguer, and L. Bordes, "Condition-based inspection/replacement policies for non-monotone deteriorating systems with environmental covariates," Reliability Engineering \& System Safety, vol. 95, no. 8, pp. 921-934, 2010.

[84] H. Tijms, A first course in stochastic models. Wiley, New York, 2003.

[85] K. Atkinson, The numerical solution of integral equations of the second kind. Cambridge University Press, 1997.

[86] Z. Botev, J. Grotowski, and D. Kroese, "Kernel density estimation via diffusion," The Annals of Statistics, vol. 38, no. 5, pp. 2916-2957, 2010.

[87] C. Audet, J. Dennis, and E. John, “Analysis of generalized pattern searches,” SIAM Journal on Optimization, vol. 13, no. 3, pp. 889-903, 2002.

[88] C. Audet and W. Hare, Derivative-Free and Blackbox Optimization. Springer Series in Operations Research and Financial Engineering, Springer International Publishing, 2017.

[89] L. Maillart and S. Pollock, "The effect of failure-distribution specification-errors on maintenance costs," in Proc. of Annual Reliability and Maintainability Symposium - RAMS 1999, pp. 69-77, 1999. doi:10.1109/RAMS.1999.744099.

Khac Tuan Huynh is an Associate Professor of Control, Reliability and Maintenance Engineering at Troyes University of Technology (UTT), France, and is a member of Charles Delaunay Institute, Systems Modeling and Dependability Group. He received his M.S., and Ph.D. degrees in System Safety and Optimization from the UTT in 2008, and 2011 respectively. His current research interests include stochastic degradation modeling of control systems, prognostic and health management techniques, and maintenance decision-making, optimization, and evaluation. He has published articles in journals such as IEEE Transactions on Reliability; IEEE Transactions on Systems, Man, and Cybernetics: Systems; Reliability Engineering \& System Safety; European Journal of Operational Research; Journal of Risk and Reliability; and in many national and international conferences. One of his papers has received First Place in the 2010 Tom Fagan Reliability and Maintainability Symposium Student Paper Competition.

Antoine Grall is a Professor of Mathematics, Reliability and Maintenance Engineering at Charles Delaunay Institute - UMR 6281, CNRS (Systems Modeling and Dependability Group), Troyes University of Technology. His current research interests include stochastic degradation modeling, lifetime prognosis, reliability of deteriorating control systems and mainly maintenance modeling and optimisation. He is especially interested in condition-based and predictive dynamic maintenance policies with integration of on-line monitoring information. He is member of the Editorial Board of Reliability Engineering \& System Safety and of the Journal of Risk and Reliability.

Christophe Bérenguer is a Professor of reliability engineering and systems control and monitoring at Gipsa-Lab, Univ. Grenoble Alpes, CNRS and Grenoble Institute of Technology (Grenoble, France). From 1997 to 2011, he was a Professor at Troyes University of Technology (Troyes, France). He has served as an officer (treasurer) for the European Safety and Reliability Association - ESRA from 2005 to 2010, and he chairs the ESRA TC on Maintenance Modelling and Applications. He has been member of the Editorial 
Board of Reliability Engineering \& System Safety (2003-2016), and he is Associate Editor of the Journal of Risk and Reliability. His research interests include system health monitoring, stochastic modeling of systems deterioration, performance evaluation and optimization of dynamic predictive maintenance policies, and probabilistic safety assessment. 